ALEA, Lat. Am. J. Probab. Math. Stat. 14, 219-244 (2017)

DOI: 10.30757/ALEA.v14-13

\title{
Continuous time autoregressive moving average processes with random Lévy coefficients
}

\section{Dirk-Philip Brandes}

Ulm University

Institute of Mathematical Finance,

Helmholtzstr. 18, 89081 Ulm, Germany.

E-mail address: dirk.brandes@uni-ulm.de

URL: http://www.uni-ulm.de/mawi/finmath/people/dirk-brandes/

\begin{abstract}
We introduce a continuous time autoregressive moving average process with random Lévy coefficients, termed RC-CARMA $(p, q)$ process, of order $p$ and $q<p$ via a subclass of multivariate generalized Ornstein-Uhlenbeck processes. Sufficient conditions for the existence of a strictly stationary solution and the existence of moments are obtained. We further investigate second order stationarity properties, calculate the autocovariance function and spectral density, and give sufficient conditions for their existence.
\end{abstract}

\section{Introduction}

Let $q<p$ be non-negative integers and $L=\left(L_{t}\right)_{t \in \mathbb{R}}$ a Lévy process, i.e. a process with stationary and independent increments, càdlàg sample paths and $L_{0}=0$ almost surely, which is continuous in probability. A CARMA $(p, q)$ process $S=$ $\left(S_{t}\right)_{t \in \mathbb{R}}$ driven by $L$ is defined via

$$
S_{t}=\mathbf{b}^{\prime} \mathbf{X}_{t}, \quad t \in \mathbb{R}
$$

with $\mathbf{X}=\left(\mathbf{X}_{t}\right)_{t \in \mathbb{R}}$ a $\mathbb{C}^{p}$-valued process which is a solution to the stochastic differential equation (SDE)

$$
\mathrm{d} \mathbf{X}_{t}=A \mathbf{X}_{t} \mathrm{~d} t+\mathbf{e} \mathrm{d} L_{t}, \quad t \in \mathbb{R},
$$

Received by the editors December 12th, 2016; accepted February 18th, 2017.

2010 Mathematics Subject Classification. 60G10, 60G51, 60H05, 60H10, 62M10.

Key words and phrases. CARMA process, continuous time autoregressive moving average process, multivariate generalized Ornstein-Uhlenbeck process, Lévy process, continuous time, random coefficients, stationarity, second order stationarity. 
where

$$
A=\left[\begin{array}{ccccc}
0 & 1 & 0 & \ldots & 0 \\
0 & 0 & 1 & \ldots & 0 \\
\vdots & \vdots & \vdots & \ddots & \vdots \\
0 & 0 & 0 & \ldots & 1 \\
-a_{p} & -a_{p-1} & -a_{p-2} & \ldots & -a_{1}
\end{array}\right], \mathbf{e}=\left[\begin{array}{c}
0 \\
0 \\
\vdots \\
0 \\
1
\end{array}\right], \quad \text { and } \quad \mathbf{b}=\left[\begin{array}{c}
b_{0} \\
b_{1} \\
\vdots \\
b_{p-2} \\
b_{p-1}
\end{array}\right]
$$

with $a_{1}, \ldots, a_{p}, b_{0}, \ldots, b_{p-1} \in \mathbb{C}$ such that $b_{q} \neq 0$ and $b_{j}=0$ for $j>q$. For $p=1$ the matrix $A$ is interpreted as $A=\left(-a_{1}\right)$.

It is well-known that the solution of (1.2) is unique for any $\mathbf{X}_{0}$ and given by

$$
\mathbf{X}_{t}=\mathrm{e}^{A t}\left(\mathbf{X}_{0}+\int_{(0, t]} \mathrm{e}^{-A s} \mathbf{e d} L_{s}\right), \quad t \geq 0
$$

Processes of this kind were first considered for $L$ being a Gaussian process by Doob (1944). Brockwell (2001b) gave the now commonly used definition with $L$ being a Lévy process.

A CARMA process $S=\left(S_{t}\right)_{t \in \mathbb{R}}$ as defined in (1.1) and (1.2) can be interpreted as a solution of the $p^{\text {th }}$-order linear differential equation

$$
a(D) S_{t}=b(D) D L_{t}
$$

where $a(z)=z^{p}+a_{1} z^{p-1}+\cdots+a_{p}, b(z)=b_{0}+b_{1} z+\cdots+b_{p-1} z^{p-1}$ and $D$ denotes the differentiation operator. In this sense, CARMA processes are a natural continuous time analog of discrete time ARMA processes. Similar to ARMA processes, CARMA processes provide a tractable but rich class of stochastic processes. Their possible autocovariance functions $h \mapsto \operatorname{Cov}\left(S_{t}, S_{t+h}\right)$ are linear combinations of (complex) exponentials and thus provide a wide variety of possible models when modeling empirical data.

In discrete time, ARMA processes with random coefficients (RC-ARMA) have attracted a lot of interest recently, in particular, AR processes with random coefficients, see e.g. Nicholls and Quinn (1982). They have applications as non-linear models for various processes, e.g. bilinear GARCH processes introduced by Storti and Vitale (2003). RC-ARMA processes also arise as a special case of conditional heteroscedastic ARMA (CHARMA) models proposed by Tsay (1987) and are used for financial volatility processes, see e.g. He and Teräsvirta (1999), to name just a few.

As CARMA processes constitute the natural continuous time analog of ARMA processes, it is, therefore, natural to ask for CARMA processes with random coefficients. The CARMA $(1,0)$ process with random (Lévy) coefficients has already been studied. It is known as the generalized Ornstein-Uhlenbeck (GOU) process, which is obtained as the solution to the SDE

$$
\mathrm{d} X_{t}=X_{t-} \mathrm{d} \xi_{t}+\mathrm{d} L_{t}, \quad t \geq 0
$$

where $(\xi, L)=\left(\xi_{t}, L_{t}\right)_{t \geq 0}$ is a bivariate Lévy process. It has been shown by de Haan and Karandikar (1989) that GOU processes arise as the natural continuous time analog of the $\operatorname{AR}(1)$ process with random i.i.d. coefficients. By choosing $\left(\xi_{t}\right)_{t \geq 0}=\left(-a_{1} t\right)_{t \geq 0}$, the GOU process reduces to the classical Lévy-driven Ornstein-Uhlenbeck process, which is a $\operatorname{CAR}(1)$, i.e. $\operatorname{CARMA}(1,0)$ process. 
Both the Ornstein-Uhlenbeck process as well as the generalized Ornstein-Uhlenbeck process have various applications in insurance and financial mathematics, see e.g. Barndorff-Nielsen and Shephard (2001) and Klüppelberg et al. (2004).

The aim of the present paper is to introduce CARMA processes with random Lévy autoregressive coefficients of higher orders, $p \geq 1$, and to study stationarity and other natural properties. The definition of our process is done in such a way that it includes the generalized Ornstein-Uhlenbeck process for order $(1,0)$ as a special case and that it reduces to the usual CARMA process when the autoregressive Lévy coefficients are chosen to be deterministic Lévy processes, i.e. pure drift and henceforth linear functions.

The paper is organized as follows. In Section 2 we give some preparative results regarding multivariate stochastic integration, the multivariate stochastic exponential, and multivariate generalized Ornstein-Uhlenbeck processes. In Section 3 we define a CARMA process with random coefficients (RC-CARMA) and present some basic properties as well as sufficient conditions for the existence of a strictly stationary solution. Similar to Brockwell and Lindner (2015) for CARMA processes, we further show that the $\operatorname{RC}-\operatorname{CARMA}(p, 0)$ process satisfies an integral-differential equation and examine its path properties. Section 4 is concerned with the existence of moments, the autocovariance function, and the spectral density, whereby it turns out that the latter two have an interesting connection to those of CARMA processes. We end Section 4 by investigating an $\operatorname{RC}-\operatorname{CARMA}(2,1)$ process in more detail. Conclusively, in Section 5 we present some simulations.

\section{Preliminaries}

Throughout, we will always assume as given a complete probability space $(\Omega, \mathcal{F}, P)$ together with a filtration $\mathbb{F}=\left(\mathcal{F}_{t}\right)_{t \geq 0}$. By a filtration we mean a family of $\sigma$-algebras $\left(\mathcal{F}_{t}\right)_{t \geq 0}$ that is increasing, i.e. $\mathcal{F}_{s} \subset \mathcal{F}_{t}$ for all $s \leq t$. Our filtration satisfies, if not stated otherwise, the usual hypotheses, i.e. $\mathcal{F}_{0}$ contains all $P$-null sets of $\mathcal{F}$, and the filtration is right-continuous.

$\mathrm{GL}(\mathbb{R}, m)$ denotes the general linear group of order $m$, i.e. the set of all $m \times m$ invertible matrices associated with the ordinary matrix multiplication. If $A \in$ $\mathrm{GL}(\mathbb{R}, m)$, we denote with $A^{\prime}$ its transpose and with $A^{-1}$ its inverse.

For càdlàg processes $X=\left(X_{t}\right)_{t \geq 0}$ we denote with $X_{t-}$ and $\Delta X_{t}:=X_{t}-X_{t-}$ the left-limit and the jump at time $t$, respectively. A $d$-dimensional Lévy processes $L=\left(L_{t}\right)_{t \geq 0}$ can be identified by its characteristic exponent $\left(A_{L}, \gamma_{L}, \Pi_{L}\right)$ due to the Lévy-Khintchine formula, i.e. if $\mu$ denotes the distribution of $L_{1}$, then its characteristic function is, for $z \in \mathbb{R}^{d}$, given by

$\widehat{\mu}(z)=\exp \left[-\frac{1}{2}\left\langle z, A_{L} z\right\rangle+i\left\langle\gamma_{L}, z\right\rangle+\int_{\mathbb{R}^{d}}\left(\mathrm{e}^{i\langle z, x\rangle}-1-i\langle z, x\rangle \mathbf{1}_{\{|x| \leq 1\}}(x)\right) \Pi_{L}(\mathrm{~d} x)\right]$.

Here, $A_{L}$ is the Gaussian covariance matrix which is in one dimension denoted by $\sigma_{L}^{2}, \Pi_{L}$ a measure on $\mathbb{R}^{d}$ which satisfies $\Pi_{L}(\{0\})=0$ and $\int_{\mathbb{R}^{d}}\left(|x|^{2} \wedge 1\right) \Pi_{L}(\mathrm{~d} x)<\infty$, called the Lévy measure, and $\gamma_{L} \in \mathbb{R}^{d}$ a constant. Further, $|x|$ denotes the Euclidean norm of $x$.

For a detailed account of Lévy processes we refer to the book of Sato (2013). 
A matrix-valued stochastic process $X=\left(X_{t}\right)_{t>0}$ is called an $\mathbb{F}$-semimartingale or simply a semimartingale if every component $\left(X_{t}^{(i, j)}\right)_{t \geq 0}$ is a semimartingale with respect to the filtration $\mathbb{F}$.

For a semimartingale $X \in \mathbb{R}^{m \times n}$, and $H \in \mathbb{R}^{l \times m}$ and $G \in \mathbb{R}^{n \times p}$ two locally bounded predictable processes, the $\mathbb{R}^{l \times p}$-valued stochastic integral $J=\int H \mathrm{~d} X G$ is defined by its components via

$$
J^{(i, j)}=\sum_{k=1}^{n} \sum_{h=1}^{m} \int H^{(i, h)} G^{(k, j)} \mathrm{d} X^{(h, k)}
$$

It can easily be seen that also in the multivariate case the stochastic integration preserves the semimartingale property as stated, for example, in the onedimensional case in Protter (2005).

For two semimartingales $X \in \mathbb{R}^{l \times m}$ and $Y \in \mathbb{R}^{m \times n}$ the $\mathbb{R}^{l \times n}$-valued quadratic covariation $[X, Y]$ is defined by its components via

$$
[X, Y]^{(i, j)}=\sum_{k=1}^{m}\left[X^{(i, k)}, Y^{(k, j)}\right],
$$

and similar its continuous part $[X, Y]^{c}$. Then

$$
[X, Y]_{t}=[X, Y]_{t}^{c}+X_{0} Y_{0}+\sum_{0<s \leq t} \Delta X_{s} \Delta Y_{s}, \quad t \geq 0
$$

is true also for matrix-valued semimartingales.

Finally, as stated in Karandikar (1991), for two semimartingales $X, Y \in \mathbb{R}^{m \times m}$ the integration by parts formula takes the form

$$
(X Y)_{t}=\int_{0+}^{t} X_{s-} \mathrm{d} Y_{s}+\int_{0+}^{t} \mathrm{~d} X_{s} Y_{s-}+[X ., Y .]_{t}, \quad t \geq 0 .
$$

\section{The Multivariate Stochastic Exponential}

Let $X=\left(X_{t}\right)_{t \geq 0}$ be a semimartingale in $\mathbb{R}^{m \times m}$ with $X_{0}=0$. Then its left stochastic exponential $\overleftarrow{\mathcal{E}}(X)_{t}$ is defined as the unique $\mathbb{R}^{m \times m}$-valued, adapted, càdlàg solution $\left(Z_{t}\right)_{t \geq 0}$ of the integral equation

$$
Z_{t}=I+\int_{(0, t]} Z_{s-} \mathrm{d} X_{s}, \quad t \geq 0
$$

where $I \in \mathbb{R}^{m \times m}$ denotes the identity matrix. The right stochastic exponential of $X$, denoted as $\overrightarrow{\mathcal{E}}(X)_{t}$, is defined as the unique $\mathbb{R}^{m \times m}$-valued, adapted, càdlàg solution $\left(Z_{t}\right)_{t \geq 0}$ of the integral equation

$$
Z_{t}=I+\int_{(0, t]} \mathrm{d} X_{s} Z_{s-}, \quad t \geq 0 .
$$

It can be shown that both the left and the right stochastic exponential are semimartingales and that for its transpose it holds $\overleftarrow{\mathcal{E}}(X)_{t}^{\prime}=\overrightarrow{\mathcal{E}}\left(X^{\prime}\right)_{t}$. As observed by Karandikar (1991), the right and the left stochastic exponentials of a semimartingale $X$ are invertible at all times $t \geq 0$ if and only if

$$
\operatorname{det}\left(I+\Delta X_{t}\right) \neq 0 \quad \forall t \geq 0
$$

We also need the following result of Karandikar (1991). 


\section{Proposition 2.1. (Inverse of the Stochastic Exponential)}

Let $X=\left(X_{t}\right)_{t \geq 0}$ be a semimartingale with $X_{0}=0$ such that (2.3) holds. Define the semimartingale

$$
U_{t}:=-X_{t}+[X, X]_{t}^{c}+\sum_{0<s \leq t}\left(\left(I+\Delta X_{s}\right)^{-1}-I+\Delta X_{s}\right), \quad t \geq 0 .
$$

Then

$$
\overleftarrow{\mathcal{E}}(X)_{t}^{-1}=\left[\overleftarrow{\mathcal{E}}\left(U^{\prime}\right)_{t}\right]^{\prime}=\overrightarrow{\mathcal{E}}(U)_{t} \quad \forall t \geq 0
$$

and

$$
U_{t}=-X_{t}-[X, U]_{t} \quad \forall t \geq 0 .
$$

Further,

$$
\operatorname{det}\left(I+\Delta U_{t}\right) \neq 0 \quad \forall t \geq 0
$$

and $X$ can be represented by

$$
X_{t}=-U_{t}+[U, U]_{t}^{c}+\sum_{0<s \leq t}\left(\left(I+\Delta U_{s}\right)^{-1}-I+\Delta U_{s}\right), \quad t \geq 0 .
$$

Proof: For (2.5) and (2.6) see Karandikar (1991), Theorem 1. For the remaining assertions, observe that $\Delta U_{t}=\left(I+\Delta X_{t}\right)^{-1}-I$ from (2.4), so that $\operatorname{det}\left(I+\Delta U_{t}\right) \neq 0$ for all $t \geq 0$. Further, from (2.4) we obtain $[U, U]_{t}^{c}=[X, X]_{t}^{c}$. Inserting this, $\Delta U_{t}=\left(I+\Delta X_{t}\right)^{-1}-I$, and the form of $U_{t}$ from (2.4) into the right-hand side of (2.7) gives $X_{t}$ so that (2.7) is true.

\section{Multivariate Generalized Ornstein-Uhlenbeck processes}

We give a short overview of results regarding multivariate generalized OrnsteinUhlenbeck (MGOU) processes which are used throughout. MGOU processes were introduced by Behme and Lindner (2012) and further investigated in Behme (2012).

Definition 2.2. Let $(X, Y)=\left(X_{t}, Y_{t}\right)_{t \geq 0}$ be a Lévy process in $\mathbb{R}^{m \times m} \times \mathbb{R}^{m}$ such that $X$ satisfies (2.3), and let $V_{0}$ be a random variable in $\mathbb{R}^{m}$. Then the $\mathbb{R}^{m}$-valued process $V=\left(V_{t}\right)_{t \geq 0}$ given by

$$
V_{t}=\overleftarrow{\mathcal{E}}(X)_{t}^{-1}\left(V_{0}+\int_{(0, t]} \overleftarrow{\mathcal{E}}(X)_{s-} \mathrm{d} Y_{s}\right), \quad t \geq 0
$$

is called a multivariate generalized Ornstein-Uhlenbeck (MGOU) process driven by $(X, Y)$. The underlying filtration $\mathbb{F}=\left(\mathcal{F}_{t}\right)_{t \geq 0}$ is such that it satisfies the usual hypotheses and such that $(X, Y)$ is a semimartingale.

The MGOU process will be called causal or non-anticipative, if $V_{0}$ is independent of $(X, Y)$, and strictly non-causal if $V_{t}$ is independent of $\left(X_{s}, Y_{s}\right)_{0 \leq s<t}$ for all $t \geq 0$.

Remark 2.3. It follows from Behme and Lindner (2012), Theorem 3.4, that an MGOU process $V=\left(V_{t}\right)_{t \geq 0}$ with an $\mathcal{F}_{0}$-measurable $V_{0}$ solves the $\mathrm{SDE}$

$$
\mathrm{d} V_{t}=\mathrm{d} U_{t} V_{t-}+\mathrm{d} Z_{t}, \quad t \geq 0,
$$


where $U=\left(U_{t}\right)_{t \geq 0}$ is another $\mathbb{R}^{m \times m}$-valued Lévy process defined by (2.4) so that $\overleftarrow{\mathcal{E}}(X)_{t}^{-1}=\overrightarrow{\mathcal{E}}(U)_{t}$, and $Z=\left(Z_{t}\right)_{t \geq 0}$ is a Lévy process in $\mathbb{R}^{m}$ given by

$$
Z_{t}=Y_{t}+\sum_{0<s \leq t}\left(\left(I+\Delta X_{s}\right)^{-1}-I\right) \Delta Y_{s}-[X, Y]_{t}^{c}, \quad t \geq 0 .
$$

With these $U$ and $Z$ the MGOU process can also be written as

$$
V_{t}=\overrightarrow{\mathcal{E}}(U)_{t}\left(V_{0}+\int_{(0, t]} \overrightarrow{\mathcal{E}}(U)_{s-}^{-1} \mathrm{~d} Y_{s}\right), \quad t \geq 0
$$

Conversely, if $(U, Z)=\left(U_{t}, Z_{t}\right)_{t \geq 0}$ is a Lévy process in $\mathbb{R}^{m \times m} \times \mathbb{R}^{m}$ such that it holds $\operatorname{det}\left(I+\Delta U_{t}\right) \neq 0$ for all $t \geq 0$, then for every $\mathcal{F}_{0}$-measurable random vector $V_{0}$ the solution to (2.8) is an MGOU process driven by $(X, Y)$, where $X$ is given by $(2.7)$ and $Y_{t}=Z_{t}+[X, Z]_{t}$.

Convention 2.4. Observe that an MGOU process and similarly the process given by (2.9) is well-defined for any starting random vector $V_{0}$, regardless if it is $\mathcal{F}_{0^{-}}$ measurable or not. We shall hence speak of (2.9) as a solution to (2.8), regardless if $V_{0}$ is $\mathcal{F}_{0}$-measurable or not. Observe that if $V_{0}$ is chosen to be independent of $(U, Z)$ or equivalently $(X, Y)$, then the natural augmented filtration of $(U, Z)$ may be enlarged by $\sigma\left(V_{0}\right)$ such that $(U, Z)$ still remains a semimartingale, see Protter (2005) Theorem VI.2. With this enlarged filtration, $V_{0}$ is measurable.

To investigate the strict stationarity property of RC-CARMA processes later, we introduce the property of irreducibility of a class of MGOU processes as it has been done in Section 4 of Behme and Lindner (2012).

Definition 2.5. Suppose that $(X, Y)=\left(X_{t}, Y_{t}\right)_{t \geq 0}$ is a Lévy process in $\mathbb{R}^{m \times m} \times \mathbb{R}^{m}$ such that $X$ satisfies (2.3). Then an affine subspace $H$ of $\mathbb{R}^{m}$ is called invariant for the class of MGOU processes $V=\left(V_{t}\right)_{t \geq 0}$ driven by $(X, Y)$ if for all $x \in H$ the choice $V_{0}=x$ implies $V_{t} \in H$ a.s. for all $t \geq 0$.

If there exists no proper affine subspace $H$ such that, for all $x \in H, V_{0}=x$ implies $V_{t} \in H$ a.s. for all $t \geq 0$, we call the class of MGOU processes irreducible.

Irreducibility is thus a property of the considered model. By abuse of language, we will call an MGOU process irreducible if the corresponding class satisfies Definition 2.5.

\section{The RC-CARMA process}

Let $p \in \mathbb{N}$ and $C=\left(C_{t}\right)_{t \geq 0}=\left(M_{t}^{(1)}, \ldots, M_{t}^{(p)}, L_{t}\right)_{t \geq 0}$ be a Lévy process in $\mathbb{R}^{p+1}$ with $\Pi_{M^{(1)}}(\{1\})=0$. Let $b_{0}, \ldots, b_{p-1} \in \mathbb{R}$. Let $U=\left(U_{t}\right)_{t \geq 0}$ be $\mathbb{R}^{p \times p}$-valued defined by

$$
U_{t}:=\left[\begin{array}{ccccc}
0 & t & 0 & \ldots & 0 \\
0 & 0 & t & \ldots & 0 \\
\vdots & \vdots & \vdots & \ddots & \vdots \\
0 & 0 & 0 & \ldots & t \\
-M_{t}^{(p)} & -M_{t}^{(p-1)} & -M_{t}^{(p-2)} & \ldots & -M_{t}^{(1)}
\end{array}\right], \quad \mathbf{b}:=\left[\begin{array}{c}
b_{0} \\
b_{1} \\
\vdots \\
b_{p-2} \\
b_{p-1}
\end{array}\right],
$$

$\mathbf{e}:=[0, \ldots, 0,1]^{\prime}$ the $p^{t h}$ unit vector, and $q:=\max \left\{j \in\{0, \ldots, p-1\}: b_{j} \neq 0\right\}$. 
Then we call any process $R=\left(R_{t}\right)_{t \geq 0}$ which satisfies

$$
R_{t}=\mathbf{b}^{\prime} V_{t}, \quad t \geq 0
$$

where $V=\left(V_{t}\right)_{t \geq 0}$ is a solution to the SDE

$$
\mathrm{d} V_{t}=\mathrm{d} U_{t} V_{t-}+\mathbf{e} \mathrm{d} L_{t}, \quad t \geq 0
$$

an $R C$-CARMA $(p, q)$ process, i.e. a CARMA process with random Lévy coefficients. We speak of $C$ and $\mathbf{b}$ as the parameters of the RC-CARMA process.

As will be seen in Proposition 3.4 below, the assumption $\Pi_{M^{(1)}}(\{1\})=0$ implies $\operatorname{det}\left(I+\Delta U_{t}\right) \neq 0$ for all $t \geq 0$, so that $V$ is an MGOU process as in (2.8) and, as in Convention 2.4, by a solution of (3.3) we mean a process of the form (2.9) with starting random variable $V_{0}$ not necessarily $\mathcal{F}_{0}$-measurable. We shall call the process $V$ a state vector process of the RC-CARMA process $R$.

Observe that we get a classical $\operatorname{CARMA}(p, q)$ process $\left(S_{t}\right)_{t \geq 0}=\left(\mathbf{b}^{\prime} V_{t}\right)_{t \geq 0}$, although on the positive real line, by choosing $\left(M_{t}^{(1)}, \ldots, M_{t}^{(p)}\right)=\left(a_{1}, \ldots, a_{p}\right) t$ with $a_{1}, \ldots, a_{p} \in \mathbb{R}$. Further, we recognize that there is less sense in choosing the coefficients of the moving average side to be random since they are just defining the weights of the components of $V$ to form $S$.

Recall that a $\operatorname{CARMA}(p, q)$ process $S=\left(S_{t}\right)_{t \in \mathbb{R}}$ satisfies the formal $p^{t h}$-order linear differential equation

$$
a(D) S_{t}=b(D) D L_{t},
$$

where $a(z)=z^{p}+a_{1} z^{p-1}+\cdots+a_{p}, b(z)=b_{0}+b_{1} z+\cdots+b_{p-1} z^{p-1}$, and $D$ denotes the differentiation with respect to $t$. When we consider an RC-CARMA $(p, 0)$ process $\left(R_{t}\right)_{t \geq 0}=\left(b_{0} V_{t}^{1}\right)_{t \geq 0}$ for $\left(V_{t}\right)_{t \geq 0}=\left(V_{t}^{1}, \ldots, V_{t}^{p}\right)_{t \geq 0}$ solving (3.3), we formally find that

$$
a_{M}(D) R_{t}=a_{M}(D) \mathbf{b}^{\prime} V_{t}=a_{M}(D) b_{0} V_{t}^{1}=b_{0} D L_{t}=b(D) D L_{t},
$$

where

$$
a_{M}(z)=z^{p}+\frac{\mathrm{d} M_{t}^{(1)}}{\mathrm{d} t} z^{p-1}+\cdots+\frac{\mathrm{d} M_{t}^{(p)}}{\mathrm{d} t} z^{0} .
$$

Observe that $\frac{\mathrm{d} M_{t}^{(i)}}{\mathrm{d} t}$ is not defined in a rigorous way but just an intuitive way of writing.

Thus, it is possible to interpret also the $\operatorname{RC}-\operatorname{CARMA}(p, 0)$ process as a solution to a formal $p^{t h}$-order linear differential equation with random coefficients.

To justify (3.5), look at the first $p-1$ components of $V$

$$
\mathrm{d} V_{t}^{i}=V_{t}^{i+1} \mathrm{~d} t \Leftrightarrow \frac{\mathrm{d} V_{t}^{i}}{\mathrm{~d} t}=V_{t}^{i+1} \Leftrightarrow D^{i} V_{t}^{1}=V_{t}^{i+1}, \quad i=1, \ldots, p-1 .
$$


Formal division by $\mathrm{d} t$ yields for the $p^{\text {th }}$ component

$$
\begin{aligned}
\mathrm{d} V_{t}^{p} & =-V_{t}^{1} \mathrm{~d} M_{t}^{(p)}-\cdots-V_{t}^{p} \mathrm{~d} M_{t}^{(1)}+\mathrm{d} L_{t} \\
“ \Leftrightarrow ” \quad \frac{\mathrm{d} V_{t}^{p}}{\mathrm{~d} t} & =-V_{t}^{1} \frac{\mathrm{d} M_{t}^{(p)}}{\mathrm{d} t}-\cdots-V_{t}^{p} \frac{\mathrm{d} M_{t}^{(1)}}{\mathrm{d} t}+\frac{\mathrm{d} L_{t}}{\mathrm{~d} t} \\
\stackrel{(3.6)}{\Leftrightarrow} \quad D V_{t}^{p} & =-V_{t}^{1} \frac{\mathrm{d} M_{t}^{(p)}}{\mathrm{d} t}-\cdots-D^{p-1} V_{t}^{1} \frac{\mathrm{d} M_{t}^{(1)}}{\mathrm{d} t}+D L_{t} \\
\Leftrightarrow & D L_{t}=D^{p} V_{t}^{1}+\frac{\mathrm{d} M_{t}^{(1)}}{\mathrm{d} t} D^{p-1} V_{t}^{1}+\cdots+\frac{\mathrm{d} M_{t}^{(p)}}{\mathrm{d} t} V_{t}^{1} \\
\Leftrightarrow & D L_{t}=a_{M}(D) V_{t}^{1} .
\end{aligned}
$$

Brockwell and Lindner (2015) gave a rigorous interpretation of (3.4) by showing that a CARMA $(p, q)$ process $\left(S_{t}=\mathbf{b}^{\prime} \mathbf{X}_{t}\right)_{t \in \mathbb{R}}$ driven by a Lévy process $L$ satisfies the integral equation

$$
a(D) J^{p} S_{t}=b(D) J^{p-1}\left(L_{t}\right)+a(D) J^{p}\left(\mathbf{b}^{\prime} \mathrm{e}^{A t} \mathbf{X}_{0}\right), \quad t \in \mathbb{R},
$$

where $a(z)$ and $b(z)$ are as before, and $J$ denotes the integration operator which associates with any càdlàg function $f=\left(f_{t}\right)_{t \in \mathbb{R}}: \mathbb{R} \rightarrow \mathbb{C}, t \mapsto f_{t}$, the function $J(f)$ defined by

$$
J(f)_{t}:=\int_{0}^{t} f_{s} \mathrm{~d} s
$$

Similarly, we give a rigorous interpretation of (3.5) as an integral-differential equation and show that the RC-CARMA $(p, 0)$ process solves this equation, hence making the formal deviation of (3.5) above thoroughly.

We call a function $g:[0, \infty) \rightarrow \mathbb{R}$ differentiable with càdlàg derivative $D g$, if $g$ is continuous and there exists a càdlàg function $D g$ such that $g$ is at every point $t \in \mathbb{R}$ right- and left-differentiable with right-derivative $D g_{t}$ and left-derivative $D g_{t-}=\lim _{\varepsilon \downarrow 0, \varepsilon \neq 0} D g_{t-\varepsilon}$, respectively. In other words, $g$ is absolutely continuous and has càdlàg density $D g$ (see the discussion in Brockwell and Lindner, 2015 at the beginning of Section 2).

We call a function $g:[0, \infty) \rightarrow \mathbb{R}$ p-times continuously differentiable with càdlàg derivative $D^{p} g$, if $g$ is $p$-1-times differentiable in the usual sense and the $(p-1)^{s t}$ derivative $D^{(p-1)} g$ is differentiable with càdlàg derivative $D^{p} g=D\left(D^{p-1} g\right)$ as defined above.

Theorem 3.1. Let $C=\left(C_{t}\right)_{t \geq 0}=\left(M_{t}^{(1)}, \ldots, M_{t}^{(p)}, L_{t}\right)_{t \geq 0}$ be a Lévy process in $\mathbb{R}^{p+1}$ and an $\mathbb{F}$-semimartingale with $\Pi_{M^{(1)}}(\{1\})=0$. Let $\mathbf{\mathbf { b }}^{\prime}=\left[b_{0}, \ldots, b_{p-1}\right] \in \mathbb{R}^{p}$ with $b_{0} \neq 0$ and $b_{1}=\cdots=b_{p-1}=0$, and consider the $R C$-CARMA $(p, 0)$ process $R=\left(R_{t}\right)_{t \geq 0}$ defined by (3.2) and (3.3), where $V=\left(V_{t}\right)_{t \geq 0}$ is the state vector process (with $V_{0}$ not necessarily $\mathcal{F}_{0}$-measurable). Denote by $\mathcal{D}^{p-1}$ the set of all $\mathbb{F}$ adapted, $\mathbb{R}$-valued processes $G=\left(G_{t}\right)_{t \geq 0}$ which are $p-1$ times differentiable with càdlàg derivative $D^{p-1} G$.

(a) Define $W=\left(W_{t}\right)_{t \geq 0}$ by

$$
W_{t}:=R_{t}-\mathbf{b}^{\prime} \overrightarrow{\mathcal{E}}(U)_{t} V_{0}, \quad t \geq 0
$$


Then $W \in \mathcal{D}^{p-1}$, it is an $R C$-CARMA process with parameters $C, \mathbf{b}$, and initial state vector $\mathbf{0}$, and it satisfies the integral-differential equation

$$
D^{p-1} W_{t}+\left(\sum_{i=1}^{p} \int_{(0, t]} D^{i-1} W_{s-} \mathrm{d} M_{s}^{(p-i+1)}\right)=b_{0} L_{t}, \quad t \geq 0 .
$$

If $V_{0}$ is additionally $\mathcal{F}_{0}$-measurable, then also $R \in \mathcal{D}^{p-1}$ and there exists an $\mathcal{F}_{0^{-}}$ measurable random variable $Z_{0}$ such that

$$
D^{p-1} R_{t}+\left(\sum_{i=1}^{p} \int_{(0, t]} D^{i-1} R_{s-} \mathrm{d} M_{s}^{(p-i+1)}\right)=b_{0} L_{t}+Z_{0}, \quad t \geq 0 .
$$

(b) Conversely, if $\widetilde{R}=\left(\widetilde{R}_{t}\right)_{t \geq 0} \in \mathcal{D}^{p-1}$ satisfies

$$
D^{p-1} \widetilde{R}_{t}+\left(\sum_{i=1}^{p} \int_{(0, t]} D^{i-1} \widetilde{R}_{s-} \mathrm{d} M_{s}^{(p-i+1)}\right)=b_{0} L_{t}+Z_{0}
$$

for some $\mathcal{F}_{0}$-measurable $Z_{0}$, then $\widetilde{R}$ is an $R C$-CARMA process with parameters $C$, b, and state vector process $\widetilde{V}=\left(\widetilde{V}_{t}\right)_{t \geq 0}:=\left(b_{0}^{-1}\left(\widetilde{R}_{t}, D \widetilde{R}_{t}, \ldots, D^{p-1} \widetilde{R}_{t}\right)\right)_{t \geq 0}$. Especially, $\widetilde{V}_{0}$ is $\mathcal{F}_{0}$-measurable.

Proof: (a) As already observed (and to be shown in Proposition 3.4 (a) below), the condition $\Pi_{M^{(1)}}(\{1\})=0$ implies that $V$ is an MGOU process. So

$$
V_{t}=\overrightarrow{\mathcal{E}}(U)_{t}\left(V_{0}+\int_{(0, t]} \overrightarrow{\mathcal{E}}(U)_{s-}^{-1} \mathrm{~d} Y_{s}\right)
$$

for some Lévy process $Y$ as specified in Remark 2.3. Hence, $\left(V_{t}-\overrightarrow{\mathcal{E}}(U)_{t} V_{0}\right)_{t \geq 0}$ is adapted and consequently so is $\left(R_{t}-\mathbf{b}^{\prime} \overrightarrow{\mathcal{E}}(U)_{t} V_{0}\right)_{t \geq 0}$, and it is obviously an RCCARMA process with initial state vector $\mathbf{0}$. Hence, for the proof of (3.7) it suffices to assume that $V_{0}=\mathbf{0}$.

Denote $V=\left(V_{t}\right)_{t \geq 0}=\left(V_{t}^{1}, \ldots, V_{t}^{p}\right)_{t \geq 0}$. By (3.6), we have $D^{i} V_{t}^{1}=V_{t}^{i+1}$, $i=1, \ldots, p-1$. Hence, $V_{t}^{1}$ is $p-1$-times differentiable with $(p-1)^{s t}$ càdlàg derivative $V_{t}^{p}$. By the defining SDE of the RC-CARMA process (3.3) and the form of the matrix $U=\left(U_{t}\right)_{t \geq 0}$ given in (3.1), we also have

$$
V_{t}^{p}=V_{0}^{p}-\sum_{i=1}^{p} \int_{(0, t]} V_{s-}^{i} \mathrm{~d} M_{s}^{(p-i+1)}+L_{t},
$$

and, since $V_{0}=0$, that

$$
L_{t}=D^{p-1} V_{t}^{1}+\sum_{i=1}^{p} \int_{(0, t]} D^{i-1} V_{s-}^{1} \mathrm{~d} M_{s}^{(p-i+1)} .
$$

Multiplying (3.11) by $b_{0}$ gives (3.7) when $V_{0}=\mathbf{0}$ and hence (3.7) in general.

For (3.8) let

$$
K_{t}=\left(K_{t}^{1}, \ldots, K_{t}^{p}\right)^{\prime}:=\overrightarrow{\mathcal{E}}(U)_{t} V_{0}
$$

When we consider the SDE which defines the right stochastic exponential $\mathrm{d} \overrightarrow{\mathcal{E}}(U)_{t}=$ $\mathrm{d} U_{t} \overrightarrow{\mathcal{E}}(U)_{t-}$, we obtain

$$
\mathrm{d} K_{t}=\mathrm{d} U_{t} K_{t-} \quad \text { with } \quad K_{0}=V_{0},
$$


and, due to the form of the process $U$,

$$
\mathrm{d} K_{t}^{i}=K_{t}^{i+1} \mathrm{~d} t \quad \Leftrightarrow \quad \frac{\mathrm{d} K_{t}^{i}}{\mathrm{~d} t}=K_{t}^{i+1} \quad \Leftrightarrow \quad D^{i} K_{t}^{1}=K_{t}^{i+1}, \quad i=1, \ldots, p-1 .
$$

Further, from the last component of (3.12)

$$
\begin{aligned}
K_{t}^{p}=D^{p-1} K_{t}^{1} & =V_{0}^{p}-\int_{(0, t]} K_{s-}^{1} \mathrm{~d} M_{s}^{p}-\cdots-\int_{(0, t]} K_{s-}^{p} \mathrm{~d} M_{s}^{1} \\
& =V_{0}^{p}-\sum_{i=1}^{p} \int_{(0, t]} D^{i-1} K_{s-}^{1} \mathrm{~d} M_{s}^{(p-i+1)} .
\end{aligned}
$$

Hence,

$$
D^{p-1} K_{t}^{1}+\sum_{i=1}^{p} \int_{(0, t]} D^{i-1} K_{s-}^{1} \mathrm{~d} M_{s}^{(p-i+1)}=V_{0}^{p}
$$

where $V_{0}^{p}$ is $\mathcal{F}_{0}$-measurable such that we obtain (3.8) via $R_{t}=W_{t}+\mathbf{b}^{\prime} K_{t}$, where $W_{t}$ satisfies (3.7).

(b) For the converse, let $\widetilde{R} \in \mathcal{D}^{p-1}$ satisfy (3.9), and denote $\widetilde{V}_{t}=\left(\widetilde{V}_{t}^{1}, \ldots, \widetilde{V}_{t}^{p}\right)=$ $\left(b_{0}^{-1}\left(\widetilde{R}_{t}, D \widetilde{R}_{t}, \ldots, D^{p-1} \widetilde{R}_{t}\right)\right), t \geq 0$. By the fundamental theorem of calculus

$$
\widetilde{V}_{t}^{i}=\widetilde{V}_{0}^{i}+\int_{(0, t]} \widetilde{V}_{s}^{i+1} \mathrm{~d} s, \quad i=1, \ldots, p-1,
$$

and from (3.9) we obtain

$$
\begin{aligned}
\widetilde{V}_{t}^{p} & =b_{0}^{-1} Z_{0}-\sum_{i=1}^{p} \int_{(0, t]} D^{i-1} \widetilde{V}_{s-}^{1} \mathrm{~d} M_{s}^{(p-i+1)}+L_{t} \\
& =b_{0}^{-1} Z_{0}-\sum_{i=1}^{p} \int_{(0, t]} \widetilde{V}_{s-}^{i} \mathrm{~d} M_{s}^{(p-i+1)}+L_{t}, \quad t \geq 0 .
\end{aligned}
$$

But (3.13) and (3.14) mean that $\widetilde{V}_{0}^{p}=b_{0}^{-1} Z_{0}$ and that $\widetilde{V}=\left(\widetilde{V}_{t}\right)_{t \geq 0}$ satisfies

$$
\widetilde{V}_{t}=\widetilde{V}_{0}+\int_{(0, t]} \mathrm{d} U_{s} \widetilde{V}_{s-}+\mathbf{e} L_{t}
$$

Since obviously $\mathbf{b}^{\prime} \widetilde{V}_{t}=b_{0} b_{0}^{-1} \widetilde{R}_{t}=\widetilde{R}_{t}$, it follows that $\widetilde{R}$ is an $\operatorname{RC-CARMA}(p, 0)$ process with parameters $C, \mathbf{b}$, and state vector process $\widetilde{V}$.

Remark 3.2. Differentiating (3.8) formally gives

$$
D^{p} R_{t}+\sum_{i=1}^{p} D^{i-1} R_{s-} D M_{s}^{(p-i+1)}=b_{0} D L_{t},
$$

hence (3.5), and the RC-CARMA $(p, 0)$ process can be interpreted as a solution to a formal $p^{\text {th }}$-order linear differential equation with random coefficients. To obtain a similar equation and hence interpretation for $\operatorname{RC}-\operatorname{CARMA}(p, q)$ processes with $q>0$ seems not so easy since it is in general not possible to interchange the stochastic integration with the differentiation operator $D$.

Remark 3.3. Similar as in case of $\operatorname{CARMA}(p, q)$ processes, we easily see for an $\operatorname{RC}$-CARMA $(p, q)$ process $R=\left(R_{t}\right)_{t \geq 0}$ with $q<p, b_{q} \neq 0$, and $b_{j}=0$ for $j>q$ with

$$
R_{t}=\mathbf{b}^{\prime} V_{t}=b_{0} V_{t}^{1}+\cdots+b_{q} V_{t}^{q+1}
$$


that, by (3.6), $R$ is $(p-q-1)$-times differentiable with $(p-q-1)^{s t}$ càdlàg derivative

$$
D^{p-q-1} R_{t}=b_{0} D^{p-q-1} V_{t}^{1}+\cdots+b_{q} D^{p-q-1} V_{t}^{q+1}=b_{0} V_{t}^{p-q}+\cdots+b_{q} V_{t}^{p} .
$$

The following proposition shows that the state vector process $V=\left(V_{t}\right)_{t \geq 0}$ is an MGOU process and gives its specific driving Lévy process $(X, Y)$. Further, $V$ is an irreducible MGOU process if we assume that $U$ is independent of $L$ and $L$ is not deterministic.

Proposition 3.4. Let $C=\left(C_{t}\right)_{t \geq 0}=\left(M_{t}^{(1)}, \ldots, M_{t}^{(p)}, L_{t}\right)_{t \geq 0}$ be an $\mathbb{R}^{p+1}$-valued Lévy process and an $\mathbb{F}$-semimartingale. Let $\Pi_{M^{(1)}}$ denote the Lévy measure of $M^{(1)}$ and let $U$ be defined as in (3.1).

(a) Then $\overleftarrow{\mathcal{E}}(U)_{t} \in \mathrm{GL}(\mathbb{R}, p)$ for all $t \geq 0$ if and only if $\Pi_{M^{(1)}}(\{1\})=0$. In this case, for any starting random vector $V_{0}$ the solution to

$$
\mathrm{d} V_{t}=\mathrm{d} U_{t} V_{t-}+\mathbf{e} \mathrm{d} L_{t}
$$

is an $M G O U$ process driven by $(X, Y)$, i.e. $V=\left(V_{t}\right)_{t \geq 0}$ takes the form

$$
V_{t}=\overleftarrow{\mathcal{E}}(X)_{t}^{-1}\left(V_{0}+\int_{(0, t]} \overleftarrow{\mathcal{E}}(X)_{s-} \mathrm{d} Y_{s}\right)=\overrightarrow{\mathcal{E}}(U)_{t}\left(V_{0}+\int_{(0, t]} \overrightarrow{\mathcal{E}}(U)_{s-}^{-1} \mathrm{~d} Y_{s}\right)
$$

for $t \geq 0$. Here, $(X, Y)=\left(X_{t}, Y_{t}\right)_{t \geq 0}$ is a Lévy process defined by

$$
X_{t}=\left[\begin{array}{ccccc}
0 & -t & 0 & \ldots & 0 \\
0 & 0 & -t & \ldots & 0 \\
\vdots & \vdots & \vdots & \ddots & \vdots \\
0 & 0 & 0 & \ldots & -t \\
N_{t}^{(p)} & N_{t}^{(p-1)} & N_{t}^{(p-2)} & \ldots & N_{t}^{(1)}
\end{array}\right]
$$

with

$$
N_{t}^{(i)}=M_{t}^{(i)}+t \sigma_{M^{(1)}, M^{(i)}}+\sum_{0<s \leq t} \frac{\Delta M_{s}^{(i)} \Delta M_{s}^{(1)}}{1-\Delta M_{s}^{(1)}}, \quad i=1, \ldots, p,
$$

where $\sigma_{M^{(1)}, M^{(i)}}$ denotes the Gaussian covariance of $M^{(1)}$ and $M^{(i)}$. X satisfies $\operatorname{det}\left(I+\Delta X_{t}\right) \neq 0$ for all $t \geq 0$, and $Y_{t}=\mathbf{e} \widetilde{Y}_{t}$ with

$$
\tilde{Y}_{t}=L_{t}+t \sigma_{M^{(1)}, L}+\sum_{0<s \leq t} \frac{\Delta M_{s}^{(1)} \Delta L_{s}}{1-\Delta M_{s}^{(1)}} .
$$

(b) Denote $M=\left(M_{t}\right)_{t \geq 0}=\left(M_{t}^{(1)}, \ldots, M_{t}^{(p)}\right)_{t \geq 0}$. Assume that $M$ is independent of $L$ and $L$ not deterministic. Then the MGOU process $V$ obtained in (a) is irreducible.

Proof: (a) Since $C$ is a Lévy process and a semimartingale with respect to $\mathbb{F}$, it is clear that also $U$ as defined in (3.1) is a semimartingale and therefore also $\overleftarrow{\mathcal{E}}(U)$ is a semimartingale with respect to $\mathbb{F}$. Since $\overleftarrow{\mathcal{E}}(U)_{t}$ is non-singular for all $t \geq 0$ if 
and only if $\operatorname{det}\left(I+\Delta U_{t}\right) \neq 0$ for all $t \geq 0$, we calculate

$$
\begin{aligned}
\operatorname{det}\left(I+\Delta U_{t}\right) & =\operatorname{det}\left(\begin{array}{ccccc}
1 & 0 & \cdots & 0 & 0 \\
0 & 1 & \cdots & 0 & 0 \\
\vdots & \vdots & \ddots & \vdots & \vdots \\
0 & 0 & \cdots & 1 & 0 \\
-\Delta M_{t}^{(p)} & -\Delta M_{t}^{(p-1)} & \ldots & -\Delta M_{t}^{(2)} & 1-\Delta M_{t}^{(1)}
\end{array}\right) \\
& =\left(1-\Delta M_{t}^{(1)}\right)
\end{aligned}
$$

which shows that $\overleftarrow{\mathcal{E}}(U)_{t}$ is non-singular if and only if $\Delta M_{t}^{(1)} \neq 1$ for all $t \geq 0$ Since $M^{(1)}$ is a Lévy process, the latter is equivalent to $\Pi_{M^{(1)}}(\{1\})=0$.

By Remark 2.3, $V$ is an MGOU process driven by $(X, Y)$, where $X$ is given by (2.7) and satisfies $\operatorname{det}\left(I+\Delta X_{t}\right) \neq 0$ for all $t \geq 0$, and $Y_{t}=\mathbf{e} L_{t}+[X, \mathbf{e} L]_{t}$.

From (2.1) we obtain for the components of $[U, U]^{c}$ due to the form of $U$ in (3.1)

$$
\left([U, U]_{t}^{c}\right)^{(i, j)}=\sum_{k=1}^{p}\left[U^{(i, k)}, U^{(k, j)}\right]^{c}= \begin{cases}0, & i=1, \ldots, p-1, \\ t \sigma_{M^{(1)}, M^{(p-j+1)}}, & i=p .\end{cases}
$$

The form of $I+\Delta U_{t}$ is implicitly given in (3.15) such that

$$
\left(I+\Delta U_{t}\right)^{-1}=\left(\begin{array}{ccccc}
1 & 0 & \cdots & 0 & 0 \\
0 & 1 & \cdots & 0 & 0 \\
\vdots & \vdots & \ddots & \vdots & \vdots \\
0 & 0 & \cdots & 1 & 0 \\
\frac{\Delta M_{t}^{(p)}}{1-\Delta M_{t}^{(1)}} & \frac{\Delta M_{t}^{(p-1)}}{1-\Delta M_{t}^{(1)}} & \cdots & \frac{\Delta M_{t}^{(2)}}{1-\Delta M_{t}^{(1)}} & \frac{1}{1-\Delta M_{t}^{(1)}}
\end{array}\right)
$$

which is well-defined since $\Pi_{M^{(1)}}(\{1\})=0$. Summing up the terms according to (2.7) leads to the stated form of the processes $\left(N_{t}^{(1)}, \ldots, N_{t}^{(p)}\right)_{t \geq 0}$ and $X$.

For $Y_{t}=\mathbf{e} L_{t}+[X, \mathbf{e} L]_{t}$ we obtain with (2.1) componentwise

$$
[X, \mathbf{e} L]_{t}^{(i)}=\sum_{k=1}^{p}\left[X^{(i, k)},(\mathbf{e} L)^{(k)}\right]_{t}=\left[X^{(i, p)}, L\right]_{t}= \begin{cases}0, & i=1, \ldots, p-1, \\ {\left[N^{(1)}, L\right]_{t},} & i=p .\end{cases}
$$

Since

$$
\Delta N_{t}^{(1)}=\frac{\Delta M_{t}^{(1)}}{1-\Delta M_{t}^{(1)}},
$$

and $\left[N^{(1)}, L\right]_{t}^{c}=\left[M^{(1)}, L\right]_{t}^{c}=t \sigma_{M^{(1)}, L}$ we get the stated form of $Y$ by (2.2).

(b) Suppose that $V=\left(V_{t}\right)_{t \geq 0}=\left(V_{t}^{1}, \ldots, V_{t}^{p}\right)_{t \geq 0}$ is not irreducible. Hence, there exists an invariant affine subspace $H$ with $\operatorname{dim} H \in\{0, \ldots, p-1\}$. Then for all $x \in H$ it holds $P\left(V_{t} \in H \mid V_{0}=x\right)=1$ for all $t \geq 0$. Since $V$ is càdlàg, we obtain $P\left(V_{t} \in H \forall t \geq 0 \mid V_{0}=x\right)=1$. Further, if $H^{\prime} \supset H$ with $\operatorname{dim} H^{\prime}=p-1$, then

$$
P\left(V_{t} \in H^{\prime} \forall t \geq 0 \mid V_{0}=x\right)=1 \quad \forall x \in H .
$$

We shall show that $P\left(V_{t} \in H^{\prime} \forall t \geq 0 \mid V_{0}=x\right)<1$ for all $x \in \mathbb{R}^{p}$, hence contradicting (3.16). So assume w.l.o.g. that $\operatorname{dim} H=p-1$. Since then $H$ is a hyperplane, there exists $\lambda \in \mathbb{R}^{p}$ with $\lambda \neq 0$ and $a \in \mathbb{R}$ such that $H=\left\{y \in \mathbb{R}^{p}: \lambda^{\prime} y=a\right\}$.

Let $V_{0}=x \in H$. Let $i_{1}, \ldots, i_{k} \in\{1, \ldots, p\}$ with $i_{n} \neq i_{m}$ for all $n \neq m, \lambda_{i_{n}} \neq 0$ for all $n \in\{1, \ldots, k\}$ and $\lambda_{j}=0$ for $j \in\{1, \ldots, p\} \backslash\left\{i_{1}, \ldots, i_{k}\right\}$. W.l.o.g. assume $i_{1}<i_{2}<\cdots<i_{k}$. By the existence of an invariant affine subspace, this yields 
$\lambda_{i_{1}} V_{t}^{i_{1}}+\cdots+\lambda_{i_{k}} V_{t}^{i_{k}}=a$. This is, since $D^{i_{n}-i_{1}} V_{t}^{i_{1}}=V_{t}^{i_{n}}, n=1, \ldots, k$, equivalent to

$$
\lambda_{i_{1}} V_{t}^{i_{1}}+\lambda_{i_{2}} D^{i_{2}-i_{1}} V_{t}^{i_{1}}+\cdots+\lambda_{i_{k}} D^{i_{k}-i_{1}} V_{t}^{i_{1}}=a .
$$

But (3.17) is an inhomogeneous ordinary linear differential equation of order $i_{k}-i_{1}$. Hence, $V_{t}^{i_{1}}$ is a smooth deterministic function in $t$ and so are $V_{t}^{j}$ for all $j \in\{1, \ldots, p\}$ since $V_{t}^{j}=D^{j-1} V_{t}^{1}$. When we consider the last component of $V$, we obtain by (3.10) with $V_{0}^{p}=x^{p}$

$$
L_{t}=V_{t}^{p}+\sum_{k=1}^{p} \int_{(0, t]} V_{s-}^{k} \mathrm{~d} M_{s}^{(p-k+1)}-x_{p}
$$

But under the assumption that $M$ and $L$ are independent and $L$ is not deterministic, (3.18) cannot hold (observe that $V_{t}$ is deterministic by (3.17) when $V_{0}=x$ ). This gives the wanted contradiction and therefore that $V$ is irreducible.

Remark 3.5. We can write (3.18) using partial integration as

$$
L_{t}=V_{t}^{p}+\sum_{k=1}^{p} V_{t}^{k} M_{t}^{(p-k+1)}-\sum_{k=1}^{p} \int_{(0, t]} M_{s-}^{(p-k+1)} \mathrm{d} V_{s}^{k}-x_{p}
$$

hence $L_{t}$ is a functional of $M$. It would therefore be enough assuming that $L$ is not measurable with respect to the filtration generated by $M$ to ensure irreducibility of the state vector process $V$.

Remark 3.6. When the components $M^{(1)}, \ldots, M^{(p)}, L$ of the Lévy process $C$ in Proposition 3.4 (a) are additionally independent, then the components do not jump together almost surely and the Gaussian covariances vanish for different components so that the formulas for $N^{(1)}, \ldots, N^{(p)}$ simplify to

$$
N_{t}^{(1)}=M_{t}^{(1)}+t \sigma_{M_{t}^{(1)}}^{2}+\sum_{0<s \leq t} \frac{\left(\Delta M_{s}^{(1)}\right)^{2}}{1-\Delta M_{s}^{(1)}} \text { and } N_{t}^{(i)}=M_{t}^{(i)} \text { a.s. for } i=2, \ldots, p \text {. }
$$

Further, $Y_{t}=\mathbf{e} L_{t}$ and $X$ are independent in that case (the latter is already true when just $L$ is independent of $\left.\left(M^{(1)}, \ldots, M^{(p)}\right)\right)$.

Recall that a process is strictly stationary if its finite-dimensional distributions are shift-invariant. As in the case of the MGOU process, we can find sufficient conditions for the existence of a strictly stationary solution of the RC-CARMA SDE (3.3) and therefore a strictly stationary RC-CARMA process. Assume throughout that the used norm $\|\cdot\|$ on $\mathbb{R}^{p \times p}$ is submultiplicative.

Theorem 3.7. Let $C=\left(C_{t}\right)_{t \geq 0}=\left(M_{t}^{(1)}, \ldots, M_{t}^{(p)}, L_{t}\right)_{t \geq 0}$ be a Lévy process in $\mathbb{R}^{p+1}$ with $\Pi_{M^{(1)}}(\{1\})=0$ and a semimartingale with respect to the given filtration $\mathbb{F}$. Let $\mathbf{b}^{\prime}:=\left[b_{0}, \ldots, b_{p-1}\right] \in \mathbb{R}^{p}, q:=\max \left\{j \in\{0, \ldots, p-1\}: b_{j} \neq 0\right\}$ and $U=$ $\left(U_{t}\right)_{t \geq 0}$ be given as in (3.1). Assume that $\mathbf{E}\left[\log ^{+}\left\|U_{1}\right\|\right]<\infty$ and $\mathbf{E}\left[\log ^{+}\left|L_{1}\right|\right]<$ $\infty$.

(a) Suppose there exists a $t_{0}>0$ such that

$$
\mathbf{E}\left[\log \left\|\overleftarrow{\mathcal{E}}(U)_{t_{0}}\right\|\right]<0
$$


Then $\overleftarrow{\mathcal{E}}(U)_{t}$ converges a.s. to 0 as $t \rightarrow \infty$, and the integral $\int_{(0, t]} \overleftarrow{\mathcal{E}}(U)_{s-} \mathbf{e d} L_{s}$ converges a.s. to a finite random vector as $t \rightarrow \infty$, denoted by $\int_{0}^{\infty} \overleftarrow{\mathcal{E}}(U)_{s-} \mathbf{e} \mathrm{d} L_{s}$. Further, (3.3) admits a strictly stationary solution which is causal and unique in distribution, and is achieved by choosing $V_{0}$ to be independent of $C$ and such that $V_{0} \stackrel{d}{=} \int_{0}^{\infty} \overleftarrow{\mathcal{E}}(U)_{s-} \mathbf{e d} L_{s}$

(b) Conversely, if $V_{0}$ can be chosen independent of $C$ such that $V$ is strictly stationary, $M=\left(M_{t}^{(1)}, \ldots, M_{t}^{(p)}\right)_{t \geq 0}$ is independent of $L$ and $L$ not deterministic, then there exists a $t_{0}>0$ such that (3.19) holds.

In both cases with this choice of $V_{0}$, the $R C$-CARMA process given by $R_{t}=\mathbf{b}^{\prime} V_{t}$, $t \geq 0$, is strictly stationary, too.

Proof: (a) The assertions regarding $V$ follow from Theorem 5.4 with Remark 5.5 (b) and Theorem 5.2 (a) in Behme and Lindner (2012).

(b) Under the assumptions made, $V$ is irreducible by Proposition 3.4 (b). Therefore Theorem 5.4 of Behme and Lindner (2012) applies.

That $R$ is strictly stationary if $V$ is, is obvious.

\section{Existence of moments and second order properties}

In this section, we calculate the autocovariance function (ACVF) of an RCCARMA process and give a connection to the autocovariance function of a specific CARMA process obtained by choosing $A=\mathbf{E}\left[U_{1}\right]$. Further, we give sufficient conditions for the existence of the ACVF and the spectral density. We end this section with an exemplary investigation of the RC-CARMA $(2,1)$ process. But we start with a result which guarantees the existence of higher moments. Assume that the used norm $\|\cdot\|$ on $\mathbb{R}^{p \times p}$ is submultiplicative.

Proposition 4.1. Let $R=\left(R_{t}\right)_{t \geq 0}$ be an $R C$-CARMA $(p, q)$ process with parameters $C=\left(C_{t}\right)_{t \geq 0}=\left(M_{t}^{(1)}, \ldots, M_{t}^{(p)}, L_{t}\right)_{t \geq 0}$, $\mathbf{b}$ and strictly stationary state vector process $V=\left(V_{t}\right)_{t \geq 0}$ with $V_{0}$ independent of $C$ and $C$ a semimartingale with respect to the given filtration $\mathbb{F}$. Assume that for $\kappa>0$ we have for some $t_{0}>0$

$$
\mathbf{E}\left\|C_{1}\right\|^{\max \{\kappa, 1\}}<\infty \quad \text { and } \quad \mathbf{E}\left\|\overleftarrow{\mathcal{E}}(U)_{t_{0}}\right\|^{\kappa}<1
$$

Then $\mathbf{E}\left|R_{0}\right|^{\kappa}<\infty$, and if (4.1) holds for $\kappa=1$,

$$
\mathbf{E}\left[R_{0}\right]=b_{0} \frac{\mathbf{E}\left[L_{1}\right]}{\mathbf{E}\left[M_{1}^{(p)}\right]} .
$$

Remark 4.2. The assumption (4.1) in the previous proposition actually already implies the existence of a strictly stationary state vector process $V=\left(V_{t}\right)_{t \geq 0}$, that is unique in distribution, since $\mathbf{E}\left\|C_{1}\right\|^{\max \{\kappa, 1\}}<\infty$ obviously implies $\mathbf{E}\left[\log ^{+}\left\|U_{1}\right\|\right]<$ $\infty$ and $\mathbf{E}\left[\log ^{+}\left|L_{1}\right|\right]<\infty$, and by Jensen's inequality and (4.1) we further have

$$
\kappa \mathbf{E}\left[\log \left\|\overleftarrow{\mathcal{E}}(U)_{t_{0}}\right\|\right] \leq \log \mathbf{E}\left\|\overleftarrow{\mathcal{E}}(U)_{t_{0}}\right\|^{\kappa}<0
$$

Then Theorem 3.7 applies.

Proof of Proposition 4.1: By Remark 4.2 the strictly stationary state vector process $V$ is unique in distribution. By Proposition 3.3 in Behme (2012) we then have $\mathbf{E}\left\|V_{0}\right\|^{\kappa}<\infty$ and hence $\mathbf{E}\left|R_{0}\right|^{\kappa}<\infty$. 
Now let $\kappa=1$. Again from Behme (2012), Proposition 3.3, we know that $\mathbf{E}\left[U_{1}\right]$ is invertible and

$$
\mathbf{E}\left[V_{0}\right]=-\mathbf{E}\left[U_{1}\right]^{-1} \mathbf{e} \mathbf{E}\left[L_{1}\right] .
$$

Observe that $\mathbf{E}\left[U_{1}\right]$ is a companion matrix and it is well-known that the inverse of this is of the form

$$
\mathbf{E}\left[U_{1}\right]^{-1}=\left[\begin{array}{ccccc}
-\frac{\mathbf{E}\left[M_{1}^{(p-1)}\right]}{\mathbf{E}\left[M_{1}^{(p)}\right]} & -\frac{\mathbf{E}\left[M_{1}^{(p-2)}\right]}{\mathbf{E}\left[M_{1}^{(p)}\right]} & \ldots & -\frac{\mathbf{E}\left[M_{1}^{(1)}\right]}{\mathbf{E}\left[M_{1}^{(p)}\right]} & -\frac{1}{\mathbf{E}\left[M_{1}^{(p)}\right]} \\
1 & 0 & \ldots & 0 & 0 \\
0 & 1 & \cdots & 0 & 0 \\
\vdots & \vdots & \ddots & \vdots & \vdots \\
0 & 0 & \cdots & 1 & 0
\end{array}\right]
$$

such that

$$
\mathbf{E}\left[V_{0}\right]=\mathbf{e}_{1} \frac{\mathbf{E}\left[L_{1}\right]}{\mathbf{E}\left[M_{1}^{(p)}\right]},
$$

where $\mathbf{e}_{1}$ denotes the first unit vector in $\mathbb{R}^{p}$, and

$$
\mathbf{E}\left[R_{0}\right]=\mathbf{E}\left[\mathbf{b}^{\prime} V_{0}\right]=\mathbf{b}^{\prime} \mathbf{e}_{1} \frac{\mathbf{E}\left[L_{1}\right]}{\mathbf{E}\left[M_{1}^{(p)}\right]}=b_{0} \frac{\mathbf{E}\left[L_{1}\right]}{\mathbf{E}\left[M_{1}^{(p)}\right]} .
$$

The following proposition gives sufficient conditions for the existence of the autocovariance function of an RC-CARMA process and states its form. We denote with $\otimes$ the Kronecker product and by vec the vectorizing operator which maps a matrix $H$ form $\mathbb{R}^{p \times m}$ into $\mathbb{R}^{p m}$ stacking its columns one under another. vec ${ }^{-1}(H)$ means the inverse operation and yields $H$.

Proposition 4.3. Let $R=\left(R_{t}\right)_{t \geq 0}$ be an $R C$-CARMA(p,q) process with parameters $C=\left(C_{t}\right)_{t \geq 0}$, $\mathbf{b}$ and state vector process $V=\left(V_{t}\right)_{t \geq 0}$ with $V_{0}$ independent of $C$ and $C$ a semimartingale with respect to the given filtration $\mathbb{F}$. Suppose it holds $\mathbf{E}\left\|C_{1}\right\|^{2}<\infty$ and $\mathbf{E}\left\|V_{s}\right\|^{2}<\infty$, then, for $t \geq 0$, we have

$$
\operatorname{Cov}\left(R_{t+h}, R_{t}\right)=\mathbf{b}^{\prime} \mathrm{e}^{h \mathbf{E}\left[U_{1}\right]} \operatorname{Cov}\left(V_{t}\right) \mathbf{b} \quad \forall h \geq 0,
$$

where $\operatorname{Cov}\left(V_{t}\right)=\mathbf{E}\left[V_{t} V_{t}^{\prime}\right]-\mathbf{E}\left[V_{t}\right] \mathbf{E}\left[V_{t}^{\prime}\right]$ denotes the covariance matrix of $V_{t}$.

In particular, if $V$ is strictly stationary, (4.1) holds for $\kappa=2$ and we denote

$$
D=\mathbf{E}\left[U_{1}\right] \otimes I+I \otimes \mathbf{E}\left[U_{1}\right]+\mathbf{E}\left[U_{1} \otimes U_{1}\right]-\mathbf{E}\left[U_{1}\right] \otimes \mathbf{E}\left[U_{1}\right],
$$

then all eigenvalues of $D$ have strictly negative real parts and the matrix

$$
F=\int_{0}^{\infty} \int_{0}^{s} \mathrm{e}^{u D}\left(\mathrm{e}^{(s-u)\left(\mathbf{E}\left[U_{1}\right] \otimes I\right)}+\mathrm{e}^{(s-u)\left(I \otimes \mathbf{E}\left[U_{1}\right]\right)}\right) \mathrm{d} u \mathrm{~d} s
$$

is finite. Further, if $\mathbf{E}\left[L_{1}\right]=0$, we obtain

$$
\operatorname{Cov}\left(R_{t+h}, R_{t}\right)=\mathbf{b}^{\prime} \mathrm{e}^{h \mathbf{E}\left[U_{1}\right]} \operatorname{vec}^{-1}\left(-D^{-1} \mathbf{e}_{p^{2}}\right) \mathbf{E}\left(L_{1}^{2}\right) \mathbf{b},
$$

where $\mathbf{e}_{p^{2}}$ denotes the $\left(p^{2}\right)^{t h}$-unit vector in $\mathbb{R}^{p^{2}}$, and if $M=\left(M_{t}^{(1)}, \ldots, M_{t}^{(p)}\right)_{t \geq 0}$ and $L$ are independent, we obtain

$$
\begin{aligned}
& \operatorname{Cov}\left(R_{t+h}, R_{t}\right) \\
& =\mathbf{b}^{\prime} \mathrm{e}^{h \mathbf{E}\left[U_{1}\right]}\left(\operatorname{vec}^{-1}\left(-D^{-1} \mathbf{e}_{p^{2}} \operatorname{Var}\left(L_{1}\right)+F \mathbf{e}_{p^{2}}\left(\mathbf{E}\left[L_{1}\right]\right)^{2}\right)-\mathbf{e}_{1} \mathbf{e}_{1}^{\prime}\left(\frac{\mathbf{E}\left[L_{1}\right]}{\mathbf{E}\left[M_{1}^{(p)}\right]}\right)^{2}\right) \mathbf{b} .
\end{aligned}
$$


Proof: This follows immediately from Proposition 3.4 and the subsequent remark in Behme (2012), by observing that

$$
\begin{aligned}
\operatorname{vec}^{-1}\left(\left(\mathbf{E}\left[U_{1}\right] \otimes \mathbf{E}\left[U_{1}\right]\right)^{-1} \operatorname{vec}\left(\mathbf{E}\left[\mathbf{e} L_{1}\right] \mathbf{E}\left[\mathbf{e} L_{1}\right]^{\prime}\right)\right) & =\mathbf{E}\left[U_{1}\right]^{-1} \mathbf{E}\left[\mathbf{e} L_{1}\right] \mathbf{E}\left[\mathbf{e} L_{1}\right]^{\prime}\left(\mathbf{E}\left[U_{1}\right]^{-1}\right)^{\prime} \\
& =\mathbf{e}_{1} \mathbf{e}_{1}^{\prime}\left(\frac{\mathbf{E}\left[L_{1}\right]}{\mathbf{E}\left[M_{1}^{(p)}\right]}\right)^{2}
\end{aligned}
$$

is obtained by (4.2), and the properties of the vectorizing and the Kronecker product operations.

The following is Remark 3.5 (a) in Behme (2012) for our purposes.

Remark 4.4. Let $C=\left(C_{t}\right)_{t \geq 0}=\left(M_{t}^{(1)}, \ldots, M_{t}^{(p)}, L_{t}\right)_{t \geq 0}$ be a Lévy process in $\mathbb{R}^{p+1}$ with $\mathbf{E}\left\|C_{1}\right\|^{2}<\infty$ satisfying $\Pi_{M^{(1)}}(\{1\})=0$ and $U=\left(U_{t}\right)_{t \geq 0}$ a Lévy process in $\mathbb{R}^{p \times p}$ of the form (3.1). Let $D$ be as in (4.3). Then

if and only if

$$
\mathbf{E}\left\|\overleftarrow{\mathcal{E}}(U)_{t_{0}}\right\|^{2}<1 \quad \text { for some } t_{0}>0
$$

all eigenvalues of $D$ have strictly negative real parts.

Therefore, that condition (4.1) holds for $\kappa=2$ can be replaced by

$\mathbf{E}\left\|C_{1}\right\|^{2}<\infty$ and all eigenvalues of $D$ have strictly negative real parts.

Let $R=\left(R_{t}\right)_{t \geq 0}$ be an RC-CARMA process with parameters $\mathbf{b}$ and $C=$ $\left(M^{(1)}, \ldots, M^{(p)}, L\right)$. If $\mathbf{E}\left|M^{(1)}\right|, \ldots, \mathbf{E}\left|M^{(p)}\right|<\infty$, we can associate to $R$ and each vector $\mathbf{X}_{0}$ a CARMA process $S=\left(S_{t}\right)_{t \geq 0}$, given by (1.1) and (1.2) with $A=\mathbf{E}\left[U_{1}\right]$.

Each of these processes, i.e. when $\mathbf{X}_{0}$ varies over all random variables, will be called a CARMA process associated with the given $R C$-CARMA process with state vector process $\left(\mathbf{X}_{t}\right)_{t \geq 0}$. It is then interesting to compare the autocovariance function of $R$ with that of $S$ provided both are strictly stationary with finite variance.

We denote with $\oplus$ the Kronecker sum, i.e. $A \oplus A=A \otimes I+I \otimes A$.

Theorem 4.5. Let $R=\left(R_{t}\right)_{t \geq 0}$ be an $R C$-CARMA $(p, q)$ process with parameters $C=\left(C_{t}\right)_{t \geq 0}$, $\mathbf{b}$ and strictly stationary state vector process $V=\left(V_{t}\right)_{t \geq 0}$ with $V_{0}$ independent of $C$, and $C$ a semimartingale with respect to the given filtration $\mathbb{F}$. Assume that (4.1) holds for $\kappa=2$, that $\mathbf{E}\left[L_{1}\right]=0$ and denote $\widetilde{D}:=\mathbf{E}\left[U_{1}\right] \oplus \mathbf{E}\left[U_{1}\right]$. Then $\mathbf{E}\left[U_{1}\right]$ and $\widetilde{D}$ have only eigenvalues with strictly negative real parts. Further, $\mathbf{X}_{0}$ can be chosen independent of $C$ and unique in distribution such that the state vector process $\left(\mathbf{X}_{t}\right)_{t \geq 0}$ of the associated CARMA process $S=\left(S_{t}\right)_{t \geq 0}$ becomes strictly stationary with finite variance. Its autocovariance function can be expressed for all $t \geq 0$ as

$$
\operatorname{Cov}\left(S_{t+h}, S_{t}\right)=\mathbf{b}^{\prime} \mathrm{e}^{h \mathbf{E}\left[U_{1}\right]} \operatorname{vec}^{-1}\left(-\widetilde{D}^{-1} \mathbf{e}_{p^{2}}\right) \mathbf{E}\left(L_{1}^{2}\right) \mathbf{b} \quad \forall h \geq 0 .
$$

Then the autocovariance function of $S$ and $R$ differ only by a multiplicative constant. More precisely,

$$
\operatorname{Cov}\left(R_{t+h}, R_{t}\right)=\operatorname{Cov}\left(S_{t+h}, S_{t}\right) \cdot \varrho_{R C} \quad \forall t, h \geq 0,
$$

where

$$
\varrho_{R C}=1+\mathbf{e}_{p^{2}}^{\prime} B \widetilde{D}^{-1} \mathbf{e}_{p^{2}}
$$


with $B=\mathbf{E}\left[U_{1} \otimes U_{1}\right]-\mathbf{E}\left[U_{1}\right] \otimes \mathbf{E}\left[U_{1}\right]$. Furthermore, if $\operatorname{Var}\left(R_{0}\right)>0$, the autocorrelation functions of both $R$ and $S$ agree, i.e.

$$
\operatorname{Corr}\left[S_{t+h}, S_{t}\right]=\operatorname{Corr}\left[R_{t+h}, R_{t}\right] \quad \forall t, h \geq 0 .
$$

Proof: That $\mathbf{E}\left[U_{1}\right]$ has only eigenvalues with strictly negative real parts follows since (4.1), by Jensen's inequality, implies

$$
\left\|\mathbf{E}\left[\overleftarrow{\mathcal{E}}(U)_{t_{0}}\right]\right\| \leq \mathbf{E}\left\|\overleftarrow{\mathcal{E}}(U)_{t_{0}}\right\| \leq\left(\mathbf{E}\left\|\overleftarrow{\mathcal{E}}(U)_{t_{0}}\right\|^{2}\right)^{1 / 2}<1
$$

Hence, since $\mathbf{E}\left[\overrightarrow{\mathcal{E}}(U)_{t_{0}}\right]=\mathrm{e}^{t_{0} \mathbf{E}\left[U_{1}\right]}$ by Proposition 3.1 in Behme (2012) we obtain by the submultiplicativity of the norm

$$
\left\|\mathrm{e}^{n t_{0} \mathbf{E}\left[U_{1}\right]}\right\| \leq\left\|\mathrm{e}^{t_{0} \mathbf{E}\left[U_{1}\right]}\right\|^{n} \leq\left(\mathbf{E}\left\|\overrightarrow{\mathcal{E}}(U)_{t_{0}}\right\|^{2}\right)^{n / 2} \rightarrow 0, \quad n \rightarrow \infty
$$

so that all eigenvalues of $\mathbf{E}\left[U_{1}\right]$ have strictly negative real parts (e.g. Proposition 11.8.2 in Bernstein, 2009). That then also $\widetilde{D}$ has only eigenvalues with strictly negative real parts follows by Fact 11.17.11 of Bernstein (2009).

That $\mathbf{X}$ admits a strictly stationary solution which is unique in distribution with finite variance and $\mathbf{X}_{0}$ independent of $C$ under the given conditions, is well-known (e.g. Brockwell, 2001a) or follows alternatively from Remark 4.2. (4.5) follows from Proposition 4.3. (4.8) is clearly true as long as (4.6) holds and $\operatorname{Var}\left(R_{0}\right) \neq 0$.

To show that (4.6) is indeed true, we recognize first that the covariance of the CARMA process $S$ and the RC-CARMA process $R$ differ only in the matrices $D$ as defined in (4.3) and $\widetilde{D}$. So, it is enough to show that

$$
x \varrho_{R C}=\widetilde{x}
$$

where $x:=D^{-1} \mathbf{e}_{p^{2}}$ and $\widetilde{x}:=\widetilde{D}^{-1} \mathbf{e}_{p^{2}}$ and $\varrho_{R C}$ is defined by (4.7).

Since both $D$ and $\widetilde{D}$, under the assumptions made, are invertible, $x$ and $\widetilde{x}$ are well-defined. Further, we have that

$$
D=\widetilde{D}+\mathbf{E}\left[U_{1} \otimes U_{1}\right]-\mathbf{E}\left[U_{1}\right] \otimes \mathbf{E}\left[U_{1}\right]=: \widetilde{D}+B,
$$

where the matrix $B=\left(b_{i, j}\right)_{i, j=1, \ldots, p^{2}}$ does only have values different from zero in the last row. The latter can be seen due to the form of the matrix $U_{1}$ by

$\mathbf{E}\left[U_{1} \otimes U_{1}\right]=\left[\begin{array}{ccccc}\mathbf{0}_{p} & \mathbf{E}\left[U_{1}\right] & \mathbf{0}_{p} & \ldots & \mathbf{0}_{p} \\ \mathbf{0}_{p} & \mathbf{0}_{p} & \mathbf{E}\left[U_{1}\right] & \ldots & \mathbf{0}_{p} \\ \vdots & \vdots & \vdots & \ddots & \vdots \\ \mathbf{0}_{p} & \mathbf{0}_{p} & \mathbf{0}_{p} & \ldots & \mathbf{E}\left[U_{1}\right] \\ -\mathbf{E}\left[M_{1}^{(p)} U_{1}\right] & -\mathbf{E}\left[M_{1}^{(p-1)} U_{1}\right] & -\mathbf{E}\left[M_{1}^{(p-2)} U_{1}\right] & \ldots & -\mathbf{E}\left[M_{1}^{(1)} U_{1}\right]\end{array}\right]$,

and

$\mathbf{E}\left[U_{1}\right] \otimes \mathbf{E}\left[U_{1}\right]=$

$$
\left[\begin{array}{ccccc}
\mathbf{0}_{p} & \mathbf{E}\left[U_{1}\right] & \mathbf{0}_{p} & \ldots & \mathbf{0}_{p} \\
\mathbf{0}_{p} & \mathbf{0}_{p} & \mathbf{E}\left[U_{1}\right] & \ldots & \mathbf{0}_{p} \\
\vdots & \vdots & \vdots & \ddots & \vdots \\
\mathbf{0}_{p}^{(p)} & \mathbf{0}_{p} & \mathbf{0}_{p} & \ldots & \mathbf{E}\left[U_{1}\right] \\
-\mathbf{E}\left[M_{1}^{(p)}\right] & -\mathbf{E}\left[M_{1}^{(p-1)}\right] \mathbf{E}\left[U_{1}\right] & -\mathbf{E}\left[M_{1}^{(p-2)}\right] \mathbf{E}\left[U_{1}\right] & \ldots & -\mathbf{E}\left[M_{1}^{(1)}\right] \mathbf{E}\left[U_{1}\right]
\end{array}\right] .
$$


Then

$$
\mathbf{e}_{p^{2}}=\widetilde{D} \widetilde{x}=(D-B) \widetilde{x}=D \widetilde{x}-\sum_{i=1}^{p^{2}} b_{p^{2}, i} \widetilde{x}_{i} \mathbf{e}_{p^{2}}
$$

such that

$$
D \widetilde{x}=\left(1+\sum_{i=1}^{p^{2}} b_{p^{2}, i} \widetilde{x}_{i}\right) \mathbf{e}_{p^{2}}=\left(1+\left(\mathbf{e}_{p^{2}}^{\prime} B\right) \widetilde{D}^{-1} \mathbf{e}_{p^{2}}\right) \mathbf{e}_{p^{2}}=\varrho_{R C} \mathbf{e}_{p^{2}} .
$$

Hence, $\widetilde{x}=\varrho_{R C} D^{-1} \mathbf{e}_{p^{2}}=\varrho_{R C} x$.

The following two propositions give handy sufficient conditions for (4.4) and therefore also for the existence of a strictly stationary solution by Remark 4.2.

Proposition 4.6. Suppose that $\mathbf{E}\left\|C_{1}\right\|^{2}<\infty$ and that $\mathbf{E}\left[U_{1}\right]$ has only eigenvalues with strictly negative real parts. Denote $\widetilde{D}:=\mathbf{E}\left[U_{1}\right] \oplus \mathbf{E}\left[U_{1}\right]$, and $B:=\mathbf{E}\left[U_{1} \otimes U_{1}\right]-$ $\mathbf{E}\left[U_{1}\right] \otimes \mathbf{E}\left[U_{1}\right]=\left(b_{i, j}\right)_{i, j=1, \ldots, p^{2}}$ with $b_{i, j}=0$ for all $i \neq p^{2}$ and all $j=1, \ldots, p^{2}$ and $b_{p^{2}, p(k-1)+j}=\operatorname{Cov}\left(M_{1}^{(p-k+1)}, M_{1}^{(p-j+1)}\right)$ for $k, j=1, \ldots, p$. Then the minimal singular value $\sigma_{\min }(\widetilde{D} \oplus \widetilde{D})$, which is the square root of the minimal eigenvalue of $(\widetilde{D} \oplus \widetilde{D})(\widetilde{D} \oplus \widetilde{D})^{\prime}$, is strictly positive, and if

$$
\sum_{i, j=1}^{p}\left[\operatorname{Cov}\left(M_{1}^{(i)}, M_{1}^{(j)}\right)\right]^{2}<\frac{1}{4} \sigma_{\min }(\widetilde{D} \oplus \widetilde{D})^{2},
$$

then (4.4) applies.

Proof: Assume that $\mathbf{E}\left[U_{1}\right]$ has only eigenvalues with strictly negative real parts. Then so does $\widetilde{D}=\mathbf{E}\left[U_{1}\right] \oplus \mathbf{E}\left[U_{1}\right]$ by Fact 11.17 .11 of Bernstein (2009) and hence also $\widetilde{D} \oplus \widetilde{D}$. In particular, $\widetilde{D} \oplus \widetilde{D}$ is invertible so that its minimal singular value is strictly positive. By Fact 11.18.17 of Bernstein (2009), the sum $D=\widetilde{D}+B$ has only eigenvalues with strictly negative real parts if $\|B\|_{\mathrm{F}}<1 / 2 \sigma_{\min }(\widetilde{D} \oplus \widetilde{D})$, where $\|\cdot\|_{\mathrm{F}}$ denotes the Frobenius norm. Due to the form of $B$, we see immediately that $\|B\|_{\mathrm{F}}^{2}=\sum_{j=1}^{p^{2}} b_{p^{2}, j}^{2}$, hence (4.9).

Let us denote with $\|A\|_{1}$ the column sum and with $\|A\|_{\infty}$ the row sum norm of a matrix $A$, respectively. Further, $\kappa_{1}(A)=\|A\|_{1}\left\|A^{-1}\right\|_{1}$ and $\kappa_{\infty}(A)=\|A\|_{\infty}\left\|A^{-1}\right\|_{\infty}$ denote the condition number of an invertible $A$ with respect to $\|\cdot\|_{1}$ and $\|\cdot\|_{\infty}^{\infty}$, respectively.

Proposition 4.7. Suppose that $\mathbf{E}\left\|C_{1}\right\|^{2}<\infty$ and that $\mathbf{E}\left[U_{1}\right]$ has only pairwise distinct eigenvalues with strictly negative real parts, which we denote by $\mu_{1}, \ldots, \mu_{p}$. Let $\widetilde{D}$ and $B$ be as in Proposition 4.6, denote

$$
S:=\left[\begin{array}{ccc}
1 & \ldots & 1 \\
\mu_{1} & \cdots & \mu_{p} \\
\vdots & \ddots & \vdots \\
\mu_{1}^{p-1} & \ldots & \mu_{p}^{p-1}
\end{array}\right], \quad \Lambda:=\operatorname{diag}\left(\mu_{1}, \ldots, \mu_{p}\right),
$$

and by $\operatorname{spec}(\widetilde{D})$ the set of all eigenvalues of $\widetilde{D}$. Then $\mathbf{E}\left[U_{1}\right]$ and $\widetilde{D}$ are diagonalizable, more precisely $S^{-1} \mathbf{E}\left[U_{1}\right] S=\Lambda$ and $\left(S^{-1} \otimes S^{-1}\right) \widetilde{D}(S \otimes S)=\Lambda \oplus \Lambda$. Further, 
if

$$
\begin{aligned}
& \text { (a) } \kappa_{1}(S)^{2} \cdot \max _{i, j=1, \ldots, p}\left|\operatorname{Cov}\left(M_{1}^{(i)}, M_{1}^{(j)}\right)\right|<\min _{\lambda \in \operatorname{spec}(\widetilde{D})}|\operatorname{Re}(\lambda)| \text {, or } \\
& \text { (b) } \kappa_{\infty}(S)^{2} \cdot \sum_{i, j=1}^{p}\left|\operatorname{Cov}\left(M_{1}^{(i)}, M_{1}^{(j)}\right)\right|<\min _{\lambda \in \operatorname{spec}(\widetilde{D})}|\operatorname{Re}(\lambda)| \text {, } \\
& \text { then (4.4) applies. }
\end{aligned}
$$

Proof: That $\mathbf{E}\left[U_{1}\right]$ is diagonalizable and that $S^{-1} \mathbf{E}\left[U_{1}\right] S=\Lambda$ under the assumption of pairwise distinct eigenvalues, is well-known, see, for example, Fact 5.16.4 in Bernstein (2009). Then

$$
\begin{aligned}
\left(S^{-1} \otimes S^{-1}\right) \widetilde{D}(S \otimes S) & =\left(S^{-1} \otimes S^{-1}\right)\left(\mathbf{E}\left[U_{1}\right] \otimes I+I \otimes \mathbf{E}\left[U_{1}\right]\right)(S \otimes S) \\
& =S^{-1} \mathbf{E}\left[U_{1}\right] S \otimes S^{-1} S+S^{-1} S \otimes S^{-1} \mathbf{E}\left[U_{1}\right] S \\
& =\Lambda \otimes I+I \otimes \Lambda=\Lambda \oplus \Lambda,
\end{aligned}
$$

so that $\widetilde{D}$ is diagonalizable and has only eigenvalues with strictly negative real parts. Let $r \in\{1, \infty\}$. By the Theorem of Bauer-Fike (see Theorem 7.2.2 in Golub and Van Loan, 1996) we have for $\mu$ being an eigenvalue of $D=\widetilde{D}+B$ that $\min _{\lambda \in \operatorname{spec}(\widetilde{D})}|\lambda-\mu| \leq \kappa_{r}(S \otimes S)\|B\|_{r}$. In particular, if

$$
\kappa_{r}(S \otimes S)\|B\|_{r}<\min _{\lambda \in \operatorname{spec}(\widetilde{D})}|\operatorname{Re} \lambda|,
$$

then $D$ can only have eigenvalues with strictly negative real parts. Observe that by Fact 9.9.61 in Bernstein (2009) it holds $\kappa_{r}(S \otimes S)=\|S \otimes S\|_{r}\left\|S^{-1} \otimes S^{-1}\right\|_{r}=$ $\|S\|_{r}^{2}\left\|S^{-1}\right\|_{r}^{2}=\kappa_{r}(S)^{2}$ so that the statement follows.

Remark 4.8. (a) Proposition 4.7 can also be formulated for other natural matrix norms corresponding to the $r$-norms with $r \in[1, \infty]$, i.e.

$$
\|A\|_{r}=\sup _{x \neq 0} \frac{\|A x\|_{r}}{\|x\|_{r}} .
$$

(b) For $r=\infty$ observe that Theorem 1 in Gautschi (1962) gives estimates for the condition number $\kappa_{\infty}(S)$ when $S$ has the form as in Proposition 4.7 which then gives feasible conditions for (4.4) to hold.

(c) Both Proposition 4.6 and 4.7 state that, if a strictly stationary CARMA process with finite second moments and matrix $A$ whose eigenvalues have only strictly negative real parts is given, then an RC-CARMA process with $\mathbf{E}\left[U_{1}\right]=A$ can be chosen to be strictly stationary with finite second moments, provided the variances of the $M^{(i)}$ are sufficiently small. In other words, the CARMA matrix may be slightly perturbed and still give a strictly stationary RC-CARMA process with finite second moments.

Example 4.9. Consider an RC-CARMA $(2,1)$ process under the assumption of Proposition 4.7. Denote with $\lambda_{1} \neq \lambda_{2}$ the eigenvalues of $\mathbf{E}\left[U_{1}\right]$ with strictly negative real parts. If

$$
\kappa \cdot \max _{i, j=1,2}\left|\operatorname{Cov}\left(M_{1}^{(i)}, M_{1}^{(j)}\right)\right|<\min _{\lambda \in \operatorname{spec}(\widetilde{D})}|\operatorname{Re}(\lambda)|,
$$


where

$$
\kappa=\left(\frac{\left(1+\max \left\{\left|\lambda_{1}\right|,\left|\lambda_{2}\right|\right\}\right) \cdot \max \left\{2,\left|\lambda_{1}\right|+\left|\lambda_{2}\right|\right\}}{\left|\lambda_{2}-\lambda_{1}\right|}\right)^{2},
$$

then $\widetilde{D}+B$ has only eigenvalues with strictly negative real parts.

Proof: Since $\mathbf{E}\left[U_{1}\right]$ is a companion matrix, we have

$$
S=\left[\begin{array}{cc}
1 & 1 \\
\lambda_{1} & \lambda_{2}
\end{array}\right] \text { and } S^{-1}=\left[\begin{array}{cc}
\frac{\lambda_{2}}{\lambda_{2}-\lambda_{1}} & -\frac{1}{\lambda_{2}-\lambda_{1}} \\
-\frac{\lambda_{1}}{\lambda_{2}-\lambda_{1}} & \frac{1}{\lambda_{2}-\lambda_{1}}
\end{array}\right] .
$$

Hence, straightforward calculations yield

$$
\kappa_{1}(S)=\frac{\left(1+\max \left\{\left|\lambda_{1}\right|,\left|\lambda_{2}\right|\right\}\right) \cdot \max \left\{2,\left|\lambda_{1}\right|+\left|\lambda_{2}\right|\right\}}{\left|\lambda_{2}-\lambda_{1}\right|} .
$$

Observe that $\kappa_{\infty}(S)=\kappa_{1}(S)$ such that Proposition 4.7 (b) gives a weaker sufficient condition.

Let $X=\left(X_{t}\right)_{t \in \mathbb{R}}$ be a weakly stationary real-valued stochastic process with $\mathbf{E}\left|X_{t}\right|^{2}<\infty$ for each $t \in \mathbb{R}$, then the autocovariance function of $X$ with lag $h$ is defined by

$$
\gamma_{X}(h):=\operatorname{Cov}\left(X_{t+h}, X_{t}\right)=\mathbf{E}\left[\left(X_{t+h}-\mathbf{E}\left[X_{t+h}\right]\right)\left(X_{t}-\mathbf{E}\left[X_{t}\right]\right)\right], \quad h \in \mathbb{R} .
$$

and the autocorrelation function of $X$ is

$$
\rho_{X}(h):=\frac{\gamma_{X}(h)}{\gamma_{X}(0)}=\operatorname{Corr}\left(X_{t+h}, X_{t}\right), \quad h \in \mathbb{R} .
$$

If $\gamma_{X}: \mathbb{R} \rightarrow \mathbb{R}$ is the autocovariance function of such a process $X=\left(X_{t}\right)_{t \in \mathbb{R}}$ with $\int_{\mathbb{R}}\left|\gamma_{X}(h)\right| \mathrm{d} h<\infty$, then its Fourier transform

$$
f_{X}(\omega):=\frac{1}{2 \pi} \int_{-\infty}^{\infty} \mathrm{e}^{-i \omega h} \gamma_{X}(h) \mathrm{d} h, \quad \omega \in \mathbb{R},
$$

is called the spectral density if the integral exists.

It is well-known (e.g. Brockwell, 2001a) that the spectral density of a CARMA $(p, q)$-process $S=\left(S_{t}\right)_{t \geq 0}$ of order $q<p$ is given by

$$
f_{S}(\omega)=\frac{\sigma^{2}}{2 \pi} \frac{|b(i \omega)|^{2}}{|a(i \omega)|^{2}}, \quad \omega \in \mathbb{R}
$$

with $\sigma^{2}$ being the variance of the driving Lévy process. Under the stated conditions of Theorem 4.5 we see that the spectral density of an $\operatorname{RC}-\operatorname{CARMA}(p, q)$ process $R=\left(R_{t}\right)_{t \geq 0}$ with parameters $C$ and $\mathbf{b}$ is given by

$$
f_{R}(\omega)=f_{S}(\omega) \varrho_{R C}, \quad \omega \in \mathbb{R},
$$

where $f_{S}(\omega)$ denotes the autocovariance function of the associated CARMA process with $A=\mathbf{E}\left[U_{1}\right]$ as in Theorem 4.5 and the constant $\varrho_{R C}$ is as in (4.7).

Remark 4.10. It is well-known that if $S=\left(S_{t}\right)_{t \in \mathbb{R}}$ is a weakly stationary CARMA $(p, q)$ process, then the equidistantly sampled process $S^{\Delta}=\left(S_{n \Delta}\right)_{n \in \mathbb{N}_{0}}, \Delta>0$, is a weak $\operatorname{ARMA}\left(p, q^{\prime}\right)$ process for some $q^{\prime}<p$, see e.g. Section 3 in Brockwell (2009).

Since under the conditions of Theorem 4.5 the autocovariance function of an RC$\operatorname{CARMA}(p, q)$ process $R=\left(R_{t}\right)_{t \geq 0}$ differs from that of the associated $\operatorname{CARMA}(p, q)$ 
process only by a multiplicative constant, it follows immediately that also $R^{\Delta}=$ $\left(R_{\Delta n}\right)_{n \in \mathbb{N}_{0}}$ is a weakly stationary $\operatorname{ARMA}\left(p, q^{\prime}\right)$ process for some $q^{\prime}<p$.

Next, we evaluate exemplarily the covariance structure of an RC-CARMA $(2,1)$ process under the assumption $\mathbf{E}\left[L_{1}\right]=0$.

\section{Example 4.11. (Covariance of RC-CARMA(2,1))}

Let $R=\left(R_{t}\right)_{t \geq 0}$ be an RC-CARMA $(2,1)$ process with parameters $C=\left(C_{t}\right)_{t \geq 0}=$ $\left(M_{t}^{(1)}, M_{t}^{(2)}, L_{t}\right)_{t \geq 0}$, b and strictly stationary state vector process $V=\left(V_{t}\right)_{t \geq 0}$. Let $V_{0}$ be independent of $C$ and $C$ a semimartingale with respect to the given filtration $\mathbb{F}$. Assume that $\mathbf{E}\left[L_{1}\right]=0$, that (4.1) holds for $\kappa=2$, and denote with $S=\left(S_{t}\right)_{t \geq 0}$ the associated CARMA process characterized by Theorem 4.5. Then, for all $t \geq 0$,

$$
\operatorname{Cov}\left(R_{t+h}, R_{t}\right)=\operatorname{Cov}\left(S_{t+h}, S_{t}\right) \varrho_{R C}=\mathbf{b}^{\prime} \mathrm{e}^{h \mathbf{E}\left[U_{1}\right]}\left[\begin{array}{c}
\frac{b_{0}}{\mathbf{E}\left[M_{1}^{(2)}\right]} \\
b_{1}
\end{array}\right] \frac{\mathbf{E}\left[L_{1}^{2}\right]}{2 \mathbf{E}\left[M_{1}^{(1)}\right]} \varrho_{R C}
$$

for all $h \geq 0$, where

$$
\varrho_{R C}=\frac{2 \mathbf{E}\left[M_{1}^{(1)}\right] \mathbf{E}\left[M_{1}^{(2)}\right]}{\left(2 \mathbf{E}\left[M_{1}^{(1)}\right]-\operatorname{Var}\left[M_{1}^{(1)}\right]\right) \mathbf{E}\left[M_{1}^{(2)}\right]-\operatorname{Var}\left[M_{1}^{(2)}\right]} .
$$

Proof: Under the assumptions made, an application of Theorem 4.5 yields an associated CARMA process $S$ and the first equality in (4.10). Clearly,

$$
\mathbf{E}\left[U_{1}\right]=\left[\begin{array}{cc}
0 & 1 \\
-\mathbf{E}\left[M_{1}^{(2)}\right] & -\mathbf{E}\left[M_{1}^{(1)}\right]
\end{array}\right],
$$

and the general form of $\operatorname{Cov}\left(R_{t+h}, R_{t}\right)$ is obtained from Proposition 4.3, i.e.

$$
\operatorname{Cov}\left(R_{t+h}, R_{t}\right)=\mathbf{b}^{\prime} \mathrm{e}^{h \mathbf{E}\left[U_{1}\right]} \operatorname{vec}^{-1}\left(-D^{-1} \mathbf{e}_{4}\right) \mathbf{E}\left(L_{1}^{2}\right) \mathbf{b} .
$$

Easy calculations show that

$$
D=\left[\begin{array}{cccc}
0 & 1 & 1 & 0 \\
-\mathbf{E}\left[M_{1}^{(2)}\right] & -\mathbf{E}\left[M_{1}^{(1)}\right] & 0 & 1 \\
-\mathbf{E}\left[M_{1}^{(2)}\right] & 0 & -\mathbf{E}\left[M_{1}^{(1)}\right] & 1 \\
\operatorname{Var}\left[M_{1}^{(2)}\right] & \eta & \eta & \mathbf{V a r}\left[M_{1}^{(1)}\right]-2 \mathbf{E}\left[M_{1}^{(1)}\right]
\end{array}\right]
$$

where $\eta:=\operatorname{Cov}\left[M_{1}^{(2)}, M_{1}^{(1)}\right]-\mathbf{E}\left[M_{1}^{(2)}\right]$. Let

$$
\varrho=\frac{1}{\left(2 \mathbf{E}\left[M_{1}^{(1)}\right]-\operatorname{Var}\left[M_{1}^{(1)}\right]\right) \mathbf{E}\left[M_{1}^{(2)}\right]-\operatorname{Var}\left[M_{1}^{(2)}\right]},
$$

and denote $y=\left[\begin{array}{llll}-\varrho & 0 & 0 & -\varrho \mathbf{E}\left[M_{1}^{(2)}\right]\end{array}\right]^{\prime}$. Then $D y=\mathbf{e}_{4}$ such that

$$
\operatorname{vec}^{-1}\left(-D^{-1} \mathbf{e}_{4}\right)=\operatorname{vec}^{-1}\left(-\left[\begin{array}{c}
-\varrho \\
0 \\
0 \\
-\varrho \mathbf{E}\left[M_{1}^{(2)}\right]
\end{array}\right]\right)=\left[\begin{array}{cc}
1 & 0 \\
0 & \mathbf{E}\left[M_{1}^{(2)}\right]
\end{array}\right] \varrho .
$$


Summarizing,

$$
\begin{aligned}
\operatorname{vec}^{-1}\left(-D^{-1} \mathbf{e}_{4}\right) \mathbf{b} & =\left[\begin{array}{cc}
\frac{1}{\mathbf{E}\left[M_{1}^{(2)}\right]} & 0 \\
0 & 1
\end{array}\right]\left[\begin{array}{c}
b_{0} \\
b_{1}
\end{array}\right] \frac{2 \mathbf{E}\left[M_{1}^{(1)}\right] \mathbf{E}\left[M_{1}^{(2)}\right]}{2 \mathbf{E}\left[M_{1}^{(1)}\right]} \varrho \\
& =\left[\begin{array}{c}
\frac{b_{0}}{\mathbf{E}\left[M_{1}^{(2)}\right]} \\
b_{1}
\end{array}\right] \frac{1}{2 \mathbf{E}\left[M_{1}^{(1)}\right]} \varrho_{R C},
\end{aligned}
$$

and with (4.11) we get the stated shape of (4.10).

Observe that for $M^{(1)}$ and $M^{(2)}$ being deterministic, $\varrho_{R C}=1$, hence dividing (4.10) by $\varrho_{R C}$ gives the autocovariance function of a CARMA $(2,1)$ process.

\section{Example 4.12. (Covariance of RC-CARMA(3,2))}

Let $R=\left(R_{t}\right)_{t \geq 0}$ be an RC-CARMA $(3,2)$ process with parameters $C=\left(C_{t}\right)_{t \geq 0}$, b and strictly stationary state vector process $V=\left(V_{t}\right)_{t \geq 0}$. Let $V_{0}$ be independent of $C$ and $C$ a semimartingale with respect to the given filtration $\mathbb{F}$. Assume that $\mathbf{E}\left[L_{1}\right]=0$ and (4.1) hold for $\kappa=2$. Then, for all $t, h \geq 0$,

$$
\operatorname{Cov}\left(R_{t+h}, R_{t}\right)=\mathbf{b}^{\prime} \mathrm{e}^{h \mathbf{E}\left[U_{1}\right]}\left[\begin{array}{c}
\frac{\mathbf{E}\left[M_{1}^{(1)}\right]}{\mathbf{E}\left[M_{1}^{(2)}\right]} b_{0}-b_{2} \\
b_{1} \\
\mathbf{E}\left[M_{1}^{(2)}\right] b_{2}-b_{0}
\end{array}\right] \frac{\mathbf{E}\left[L_{1}^{2}\right]}{2\left(\mathbf{E}\left[M_{1}^{(1)}\right] \mathbf{E}\left[M_{1}^{(2)}\right]-\mathbf{E}\left[M_{1}^{(3)}\right]\right)} \cdot \varrho_{R C},
$$

where

$$
\varrho_{R C}=\frac{2\left(\mathbf{E}\left[M_{1}^{(1)}\right] \mathbf{E}\left[M_{1}^{(2)}\right]-\mathbf{E}\left[M_{1}^{(3)}\right]\right)}{\xi+2\left(\operatorname{Cov}\left(M_{1}^{(3)}, M_{1}^{(1)}\right)-\mathbf{E}\left[M_{1}^{(3)}\right]\right)-\frac{\mathbf{E}\left[M_{1}^{(1)}\right]}{\mathbf{E}\left[M_{1}^{(3)}\right]} \operatorname{Var}\left[M_{1}^{(3)}\right]}
$$

with $\xi=\left(2 \mathbf{E}\left[M_{1}^{(1)}\right]-\operatorname{Var}\left[M_{1}^{(1)}\right]\right) \mathbf{E}\left[M_{1}^{(2)}\right]-\operatorname{Var}\left[M_{1}^{(2)}\right]$.

The proof follows by similar calculations as in Example 4.11 and is left to the reader.

\section{Simulations}

We compare in this section two simulations of an RC-CARMA $(2,1)$ process, one when $\mathbf{E}\left[U_{1}\right]$ has only real strictly negative eigenvalues and the other when $\mathbf{E}\left[U_{1}\right]$ has complex eigenvalues with strictly negative real parts.

For our simulations we have chosen as random coefficient processes $M^{(1)}, M^{(2)}$ two independent compound poisson processes, i.e. with depiction

$$
M_{t}^{(1)}=\sum_{i=1}^{N_{t}^{(1)}} X_{i}^{(1)} \quad \text { and } \quad M_{t}^{(2)}=\sum_{i=1}^{N_{t}^{(2)}} X_{i}^{(2)}, \quad t \geq 0 .
$$

In the case of real eigenvalues we have chosen $\mathbf{E}\left[N_{1}^{(1)}\right]=1.5, \mathbf{E}\left[N_{1}^{(2)}\right]=2, X_{i}^{(1)} \sim$ $\mathcal{N}\left(1,0.3^{2}\right)$, and $X_{i}^{(2)} \sim \mathcal{N}\left(0.25,0.2^{2}\right)$, and as driving process a standard Brownian motion $B=\left(B_{t}\right)_{t \geq 0}$. Hence, for $\widetilde{D}=\mathbf{E}\left[U_{1}\right] \oplus \mathbf{E}\left[U_{1}\right]$ and $D=\widetilde{D}+\mathbf{E}\left[U_{1} \otimes U_{1}\right]-$ 
$\mathbf{E}\left[U_{1}\right] \otimes \mathbf{E}\left[U_{1}\right]$ we have

$$
\begin{aligned}
\mathbf{E}\left[U_{1}\right]=\left[\begin{array}{cc}
0 & 1 \\
-1 / 2 & -3 / 2
\end{array}\right], & \widetilde{D}=\left[\begin{array}{cccc}
0 & 1 & 1 & 0 \\
-1 / 2 & -3 / 2 & 0 & 1 \\
-1 / 2 & 0 & -3 / 2 & 1 \\
0 & -1 / 2 & -1 / 2 & -3
\end{array}\right], \text { and } \\
D & =\left[\begin{array}{cccc}
0 & 1 & 1 & 0 \\
-1 / 2 & -3 / 2 & 0 & 1 \\
-1 / 2 & 0 & -3 / 2 & 1 \\
0.205 & -1 / 2 & -1 / 2 & -1.365
\end{array}\right] .
\end{aligned}
$$

Consequently, we have for $\mathbf{E}\left[U_{1}\right]$ the eigenvalues $\mu_{1}=-1 / 2$ and $\mu_{2}=-1$, and for $D$ the eigenvalues $\lambda_{1} \approx-0.29, \lambda_{2} \approx-1.29+1.28 i, \lambda_{3}=-1.29-1.28 i$, and $\lambda_{4}=-1.5$. Since all eigenvalues of $D$ have strictly negative real parts, we obtain the existence of a strictly stationary solution by Remark 4.2 and Remark 4.4.

Nevertheless, observe that

$$
\operatorname{Var}\left(M_{1}^{(1)}\right)+\operatorname{Var}\left(M_{1}^{(2)}\right)=1.84 \nless \frac{1}{4} \sigma_{\min }(\widetilde{D} \oplus \widetilde{D})^{2} \approx 0.0799
$$

showing that the condition in Proposition 4.6 is not necessary. Moreover,

$$
\kappa=\left(\frac{\left(1+\max \left\{\left|\lambda_{1}\right|,\left|\lambda_{2}\right|\right\}\right) \cdot \max \left\{2,\left|\lambda_{1}\right|+\left|\lambda_{2}\right|\right\}}{\left|\lambda_{2}-\lambda_{1}\right|}\right)^{2}=25
$$

so that

$$
\kappa \cdot \max _{i=1,2}\left|\operatorname{Var}\left(M_{1}^{(i)}\right)\right|=40.875 \nless \min _{\lambda \in \operatorname{spec}(\widetilde{D})}|\operatorname{Re}(\lambda)|=1,
$$

showing that also the condition in Example 4.11 and hence in Proposition 4.7 is not necessary.

In case of complex eigenvalues we have chosen $\mathbf{E}\left[N_{1}^{(1)}\right]=2, \mathbf{E}\left[N_{1}^{(2)}\right]=7.5$, $X_{i}^{(1)} \sim \mathcal{N}\left(0.5,0.1^{2}\right)$, and $X_{i}^{(2)} \sim \mathcal{N}\left(0.4,0.05^{2}\right)$, and have left the driving process unchanged. Then

$$
\mathbf{E}\left[U_{1}\right]=\left[\begin{array}{cc}
0 & 1 \\
-3 & -1
\end{array}\right]
$$

gives two complex-valued eigenvalues $\widetilde{\mu}_{1} \approx-0.5+1.66 i$ and $\widetilde{\mu}_{2}=-0.5-1.66 i$. Also, $D$ has just eigenvalues with strictly negative real parts. Furthermore, an observation similar to the one above can be made showing non-necessity of the conditions in Proposition 4.6 and 4.7.

For both the complex and the real eigenvalues case, we have simulated 10, 000, 000 observations with a mesh size $k=0.01$, i.e. $R_{0.01}, R_{0.02}, \ldots, R_{100,000}$. In Figure 5.1(a) and (c) we see the corresponding plots until time 300. Plots 5.1(b) and (d) show the corresponding autocovariance functions (ACVF).

The solid line corresponds to the model autocovariance function, the dashed one to the sampled ACVF based on the data $R_{0.01}, \ldots, R_{100,000}$, and the dotted shows the model ACVF of the corresponding $\operatorname{CARMA}(2,1)$ process. The dashed-dotted line shows the sample ACVF based on $R_{0.01}, \ldots, R_{100}$.

We see that using 10,000,000 observations to calculate the sample autocovariance function, it nearly agrees in both cases with the model autocovariance function. Plot 1(d) shows a sinusoidal oscillation which is also in the CARMA case characteristic for allowing complex eigenvalues and visualizes the variety of possible autocovariance functions. 


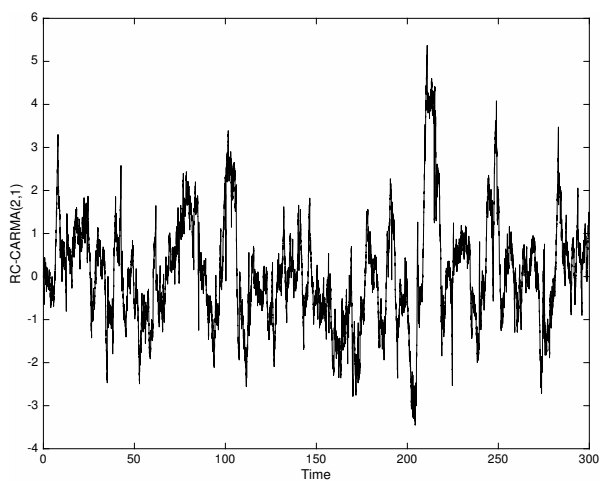

(a) Simulation with eigenvalues of $\mathbf{E}\left[U_{1}\right]$ chosen to be $\mu_{1}=-\frac{1}{2}$ and $\mu_{2}=-1$.

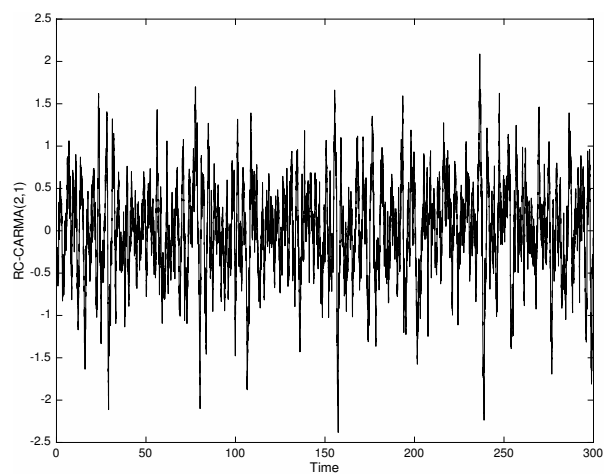

(c) Simulation with eigenvalues of $\mathbf{E}\left[U_{1}\right]$ chosen to be $\widetilde{\mu}_{1} \approx-0.5+1.66 i$ and $\widetilde{\mu}_{2}=-0.5-$ $1.66 i$.

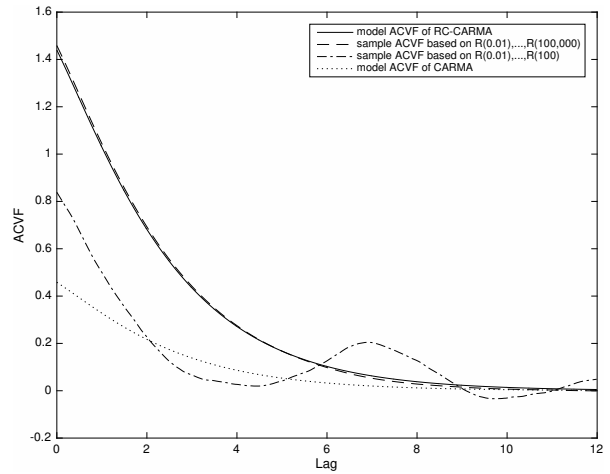

(b) ACVFs with eigenvalues of $\mathbf{E}\left[U_{1}\right]$ chosen to be $\mu_{1}=-\frac{1}{2}$ and $\mu_{2}=-1$.

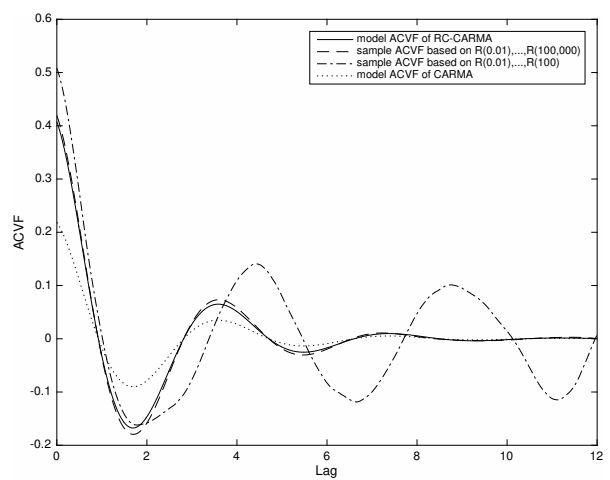

(d) ACVFs with eigenvalues of $\mathbf{E}\left[U_{1}\right]$ chosen to be $\widetilde{\mu}_{1} \approx-0.5+1.66 i$ and $\widetilde{\mu}_{2}=-0.5-1.66 i$.

Figure 5.1. Simulated RC-CARMA $(2,1)$ process and ACVFs.

Figure 5.2(a) shows all simulated observations of the RC-CARMA $(2,1)$ process where $\mathbf{E}\left[U_{1}\right]$ has real eigenvalues. On the other hand, Figure 5.2(b) shows an equally sized simulation of the associated $\operatorname{CARMA}(2,1)$ process, i.e. with the choice $A=\mathbf{E}\left[U_{1}\right]$. It can be seen that the RC-CARMA $(2,1)$ process provides larger outliers around the between -5 and 5 concentrated band than the CARMA $(2,1)$ process around its band. This may indicate possible heavy tails of RC-CARMA processes.

We justify these observations intuitively in the following remark recalling results on random recurrence equations.

Remark 5.1. Observe that a stationary CARMA process driven by a Brownian motion has a normal marginal stationary distribution, in particular, it has light tails. 


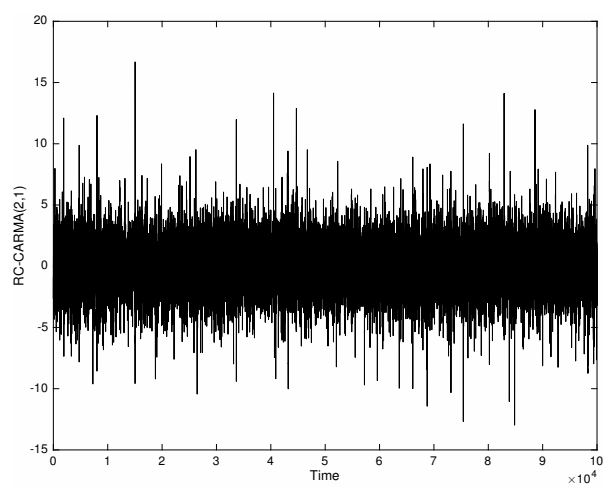

(a) Simulation of $\operatorname{RC}-\operatorname{CARMA}(2,1)$ with eigenvalues of $\mathbf{E}\left[U_{1}\right]$ chosen to be $\mu_{1}=-\frac{1}{2}$ and $\mu_{2}=-1$.

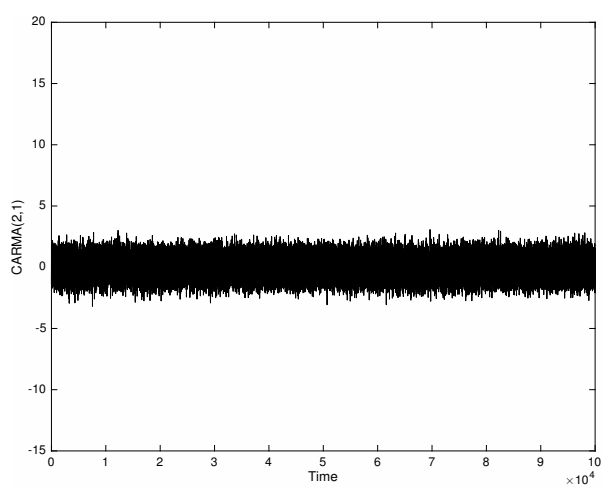

(b) Simulation of associated $\operatorname{CARMA}(2,1)$ with $A=\mathbf{E}\left[U_{1}\right]$.

Figure 5.2. Simulated RC-CARMA $(2,1)$ and CARMA $(2,1)$ processes.

On the other hand, as a consequence of results of Kesten (1973) and Goldie (1991), it is known that a generalized Ornstein-Uhlenbeck process and so an RCCARMA $(1,0)$ process will have Pareto tails under wide conditions, even if the driving process is a Brownian motion, see e.g. Lindner and Maller (2005) (Theorem 4.5) or Behme (2011) (Theorem 4.1).

We henceforth expect using the multivariate results of Kesten (1973) that under wide conditions the RC-CARMA process will also have Pareto tails for higher orders. However, we leave a thorough investigation of this for forthcoming research.

\section{Acknowledgements}

The author would like to thank an anonymous referee for pointing out several mistakes in a preliminary version. The author is very grateful to Alexander Lindner for many hours of discussion and valuable advice which greatly improved the paper.

\section{References}

O. E. Barndorff-Nielsen and N. Shephard. Modelling by Lévy processes for financial econometrics. In O.E. Barndorff-Nielsen, T. Mikosch and S. Resnick, editors, Lévy processes: Theory and Applications, pages 283-318. Birkhäuser, Boston (2001). ISBN 0-8176-4167-X. MR1833689.

A. Behme. Distributional properties of solutions of $\mathrm{d} V_{T}=V_{T}-\mathrm{d} U_{T}+\mathrm{d} L_{T}$ with Lévy noise. Adv. in Appl. Probab. 43 (3), 688-711 (2011). MR2858217.

A. Behme. Moments of MGOU processes and positive semidefinite matrix processes. J. Multivariate Anal. 111, 183-197 (2012). MR2944414.

A. Behme and A. Lindner. Multivariate generalized Ornstein-Uhlenbeck processes. Stochastic Process. Appl. 122 (4), 1487-1518 (2012). MR2914760.

D. S. Bernstein. Matrix mathematics. Princeton University Press, Princeton, NJ, second edition (2009). ISBN 978-0-691-14039-1. MR2513751. 
P. J. Brockwell. Continuous-time ARMA processes. In C. R. Rao D. N. Shanbhag, editor, Stochastic Processes: Theory and Methods, volume 19 of Handbook of Statist., pages 249-276. Elsevier, Amsterdam (2001a). MR1861726.

P. J. Brockwell. Lévy-driven CARMA processes. Ann. Inst. Statist. Math. 53 (1), 113-124 (2001b). MR1820952.

P. J. Brockwell. Lévy-driven continuous-time ARMA processes. In T. G. Andersen, R. A. Davis, J.-P. Kreiss and T. V. Mikosch, editors, Handbook Fin. Time Series, pages 457-480. Springer, Berin (2009).

P. J. Brockwell and A. Lindner. CARMA processes as solutions of integral equations. Statist. Probab. Lett. 107, 221-227 (2015). MR3412780.

J. L. Doob. The elementary Gaussian processes. Ann. Math. Statistics 15, 229-282 (1944). MR0010931.

W. Gautschi. On inverses of Vandermonde and confluent Vandermonde matrices. Numer. Math. 4, 117-123 (1962). MR0139627.

C. M. Goldie. Implicit renewal theory and tails of solutions of random equations. Ann. Appl. Probab. 1 (1), 126-166 (1991). MR1097468.

G. H. Golub and C. F. Van Loan. Matrix computations. Johns Hopkins Studies in the Mathematical Sciences. Johns Hopkins University Press, Baltimore, MD, third edition (1996). MR1417720.

L. de Haan and R. L. Karandikar. Embedding a stochastic difference equation into a continuous-time process. Stochastic Process. Appl. 32 (2), 225-235 (1989). MR1014451.

C. He and T. Teräsvirta. Properties of moments of a family of GARCH processes. J. Econometrics 92 (1), 173-192 (1999). MR1706988.

R. L. Karandikar. Multiplicative decomposition of nonsingular matrix valued semimartingales. In Séminaire de Probabilités, XXV, volume 1485 of Lecture Notes in Math., pages 262-269. Springer, Berlin (1991). MR1187784.

H. Kesten. Random difference equations and renewal theory for products of random matrices. Acta Math. 131, 207-248 (1973). MR0440724.

C. Klüppelberg, A. Lindner and R. Maller. A continuous-time GARCH process driven by a Lévy process: stationarity and second-order behaviour. J. Appl. Probab. 41 (3), 601-622 (2004). MR2074811.

A. Lindner and R. Maller. Lévy integrals and the stationarity of generalised Ornstein-Uhlenbeck processes. Stochastic Process. Appl. 115 (10), 1701-1722 (2005). MR2165340.

D. F. Nicholls and B. G. Quinn. Random coefficient autoregressive models: an introduction, volume 11 of Lecture Notes in Statistics. Springer-Verlag, New York-Berlin (1982). ISBN 0-387-90766-1. MR671255.

P. E. Protter. Stochastic integration and differential equations, volume 21 of Stochastic Modelling and Applied Probability. Springer-Verlag, Berlin (2005). ISBN 3-540-00313-4. MR2273672.

K. Sato. Lévy processes and infinitely divisible distributions, volume 68 of Cambridge Studies in Advanced Mathematics. Cambridge University Press, Cambridge (2013). ISBN 978-1-107-65649-9. MR3185174.

G. Storti and C. Vitale. BL-GARCH models and asymmetries in volatility. Stat. Methods Appl. 12 (1), 19-39 (2003). MR2081749.

R. S. Tsay. Conditional heteroscedastic time series models. J. Amer. Statist. Assoc. 82 (398), 590-604 (1987). MR898364. 